\title{
Distributed Coordinated Tracking With Reduced Interaction via a Variable Structure Approach
}

\author{
Yongcan Cao, Member, IEEE, and Wei Ren, Member, IEEE
}

\begin{abstract}
A distributed coordinated tracking problem is solved via a variable structure approach when there exists a dynamic virtual leader who is a neighbor of only a subset of a group of followers, all followers have only local interaction, and only partial measurements of the states of the virtual leader and the followers are available. In the context of coordinated tracking, we focus on both consensus tracking and swarm tracking algorithms. In the case of first-order kinematics, we propose a distributed consensus tracking algorithm without velocity measurements under both fixed and switching network topologies. In particular, we show that distributed consensus tracking can be achieved in finite time. The algorithm is then extended to achieve distributed swarm tracking without velocity measurements. In the case of second-order dynamics, we first propose two distributed consensus tracking algorithms without acceleration measurements when the velocity of the virtual leader is varying under, respectively, a fixed and switching network topology. In particular, we show that the proposed algorithms guarantee at least global exponential tracking. We then propose a distributed consensus tracking algorithm and a distributed swarm tracking algorithm when the velocity of the virtual leader is constant. When the velocity of the virtual leader is varying, distributed swarm tracking is solved by using a distributed estimator. For distributed consensus tracking, a mild connectivity requirement is proposed by adopting an adaptive connectivity maintenance mechanism in which the adjacency matrix is defined in a proper way. Similarly, a mild connectivity requirement is proposed for distributed swarm tracking by adopting a connectivity maintenance mechanism in which the potential function is defined in a proper way. Several simulation examples are presented as a proof of concept.
\end{abstract}

Index Terms-Consensus tracking, cooperative control, distributed control, multiagent systems, swarm tracking, variable structure approach.

\section{INTRODUCTION}

I N THE past two decades, multivehicle cooperative control has received significant attention in the systems and controls society. The motivation behind multivehicle cooperative control is that a group of vehicles working cooperatively can achieve great benefits including low cost, high adaptivity, and easy maintenance [2]-[6].

A distributed approach used in multivehicle cooperative control is consensus, which means that a group of vehicles reaches

Manuscript received May 01, 2009; revised October 19, 2009; accepted April 02, 2011. Date of publication April 25, 2011; date of current version December 29,2011 . Preliminary versions of the work have been presented at the 2010 American Control Conference. This work was supported by the National Science Foundation under CAREER Award ECCS-0748287. Recommended by Associate Editor M. Prandini.

The authors were with the Department of Electrical and Computer Engineering, Utah State University, Logan, UT 84322-4120 USA. They are now with the Department of Electrical Engineering, University of California, Riverside, CA 92521 USA (e-mail: ycao@ee.ucr.edu; ren@ee.ucr.edu).

Color versions of one or more of the figures in this paper are available online at http://ieeexplore.ieee.org.

Digital Object Identifier 10.1109/TAC.2011.2146830 an agreement on a common value by interacting with their local (time-varying) neighbors. Consensus has been studied for systems with both first-order kinematics and second-order dynamics. Recent study of consensus and its applications in distributed multivehicle cooperative control can be found in [7] and [8]. Existing consensus algorithms were often studied either when there does not exist a leader or when the leader is static. Although consensus without a leader is useful in applications such as cooperative rendezvous of a group of vehicles, there are many applications that require a dynamic leader. Examples include formation flying, body guard, and coordinated tracking applications.

Consensus with a dynamic leader, called consensus tracking hereafter, has been studied from different perspectives. The objective of consensus tracking is that a group of followers tracks a dynamic leader with local interaction. The authors in [9] and [10] proposed and analyzed a consensus tracking algorithm under a variable undirected network topology. However, [9] and [10] require the availability of the leader's acceleration input to all followers and/or the design of distributed observers. In [11] and [12], the authors proposed a proportional-and-derivative-like consensus tracking algorithm under a directed network topology in both continuous-time and discrete-time settings. However, [11] and [12] require either the estimates of the leader's velocity and the followers' velocities or a small sampling period. In [13], the authors studied a leader-follower consensus tracking problem with time-varying delays. However, [13] requires the velocity measurements of the followers and an estimator to estimate the leader's velocity. In addition to the consensus tracking algorithms, various flocking and swarm tracking algorithms were also studied when there exists a leader. The objective of flocking or swarm tracking with a leader is that a group of followers tracks the leader while the followers and the leader maintain a desired geometrical configuration. In [14], the author studied a flocking algorithm under the assumption that the leader's velocity is constant and is available to all followers. The authors in [15] extended the results in [14] in two aspects. When the leader has a constant velocity, [15] requires accurate position and velocity measurements of the leader. When the leader has a varying velocity, [15] requires that the leader's position, velocity, and acceleration are available to all followers. In [16], flocking of a group of autonomous vehicles with a dynamic leader was solved by using a set of switching control laws. However, [16] requires the availability of the acceleration of the leader. In [17], the authors studied a swarm tracking algorithm via a variable structure approach using artificial potentials and the sliding mode control technique. However, [17] requires the availability of the leader's position to all followers and an all-to-all communication pattern among all followers.

Taking into account the limitations in the aforementioned references, we focus on solving a distributed coordinated tracking 
problem via a variable structure approach when there exists a $d y$ namic virtual leader under the following three assumptions: 1) the virtual leader is a neighbor of only a subset of a group of followers; 2) there exists only local interaction among all followers; and 3) the velocity measurements of the virtual leader and all followers in the case of first-order kinematics or the accelerations of the virtual leader and all followers in the case of second-order dynamics are not required. In the context of this paper, we use the term coordinated tracking to refer to both consensus tracking and swarm tracking. In contrast to the assumptions that appeared in the aforementioned references, the three assumptions are more general and practical. The contributions of this paper are twofolds. In the case of first-order kinematics, we propose a distributed consensus tracking algorithm without velocity measurements under both fixed and switching network topologies. In particular, we show that distributed consensus tracking can be achieved in finite time. We then extend the result to achieve distributed swarm tracking without velocity measurements. In the case of second-order kinematics, we first propose two distributed consensus tracking algorithms without acceleration measurements when the velocity of the virtual leader is varying under, respectively, a fixed and switching network topology. In particular, we show that the proposed algorithms guarantee at least global exponential tracking. We then propose a distributed consensus tracking algorithm and a distributed swarm tracking algorithm when the velocity of the virtual leader is constant. When the velocity of the virtual leader is varying, distributed swarm tracking is solved by using a distributed estimator. For distributed consensus tracking, a mild connectivity requirement is proposed by adopting an adaptive connectivity maintenance mechanism in which the adjacency matrix is defined in a proper way. Similarly, a mild connectivity requirement is proposed for distributed swarm tracking by adopting a connectivity maintenance mechanism in which the potential function is defined in a proper way.

The remainder of this paper is organized as follows. In Section II, the graph theory notions used throughout this paper are introduced. Sections III and IV are the main parts of this paper focusing on distributed coordinated tracking for, respectively, first-order kinematics and second-order dynamics. Several simulation examples are presented in Section V. Conclusion and future works are given in Section VI.

\section{BACKGROUND AND PRELIMINARIES}

Suppose that a team consists of $n$ vehicles. We use a weighted undirected graph $\mathcal{G}=(\mathcal{V}, \mathcal{E}, \mathcal{A})$ to model the interaction among these vehicles, where $\mathcal{V}=\{1, \ldots, n\}$ is the node set, $\mathcal{E} \subseteq$ $\mathcal{V} \times \mathcal{V}$ is the edge set, and $\mathcal{A}=\left[a_{i j}\right] \in \mathbb{R}^{n \times n}$ is the weighted adjacency matrix. An edge $(i, j)$ in $\mathcal{G}$ denotes that vehicles $i$ and $j$ can obtain information from each other. Vehicle $j$ is a neighbor of vehicle $i$ if $(j, i) \in \mathcal{E}$. The weighted adjacency matrix $\mathcal{A}$ associated with $\mathcal{G}$ is defined such that $a_{i j}$ is a positive weight if $(j, i) \in \mathcal{E}$, and $a_{i j}=0$ otherwise. Note that here $a_{i j}=a_{j i}$, $\forall i \neq j$, since $(j, i) \in \mathcal{E}$ implies $(i, j) \in \mathcal{E}$.

A path is a sequence of edges in an undirected graph of the form $\left(i_{1}, i_{2}\right),\left(i_{2}, i_{3}\right), \ldots$, where $i_{j} \in \mathcal{V}$. An undirected graph is connected if there is an undirected path between every pair of distinct nodes.
Let the Laplacian matrix $\mathcal{L}=\left[\ell_{i j}\right] \in \mathbb{R}^{n \times n}$ associated with $\mathcal{A}$ be defined as $\ell_{i i}=\sum_{j=1, j \neq i}^{n} a_{i j}$ and $\ell_{i j}=-a_{i j}, i \neq j$. Note that $\mathcal{L}$ is symmetric positive semidefinite. Also note that $\mathcal{L}$ has a simple zero eigenvalue with an associated eigenvector $\mathbf{1}$, where 1 is an all-one column vector with a compatible size, and all other eigenvalues are positive if and only if $\mathcal{G}$ is connected [18].

\section{Distributed COORDINATED TRACKING FOR FIRST-ORDER KINEMATICS}

In this section, we study distributed coordinated tracking for first-order kinematics. Suppose that in addition to the $n$ vehicles, labeled as vehicles 1 to $n$, called followers hereafter, there exists a virtual leader, labeled as vehicle 0 , with a (time-varying) position $r_{0}$ and velocity $\dot{r}_{0}$. We assume that $\left|\dot{r}_{0}\right| \leq \gamma_{\ell}$, where $\gamma_{\ell}$ is a positive constant.

Consider followers with first-order kinematics given by

$$
\dot{r}_{i}=u_{i}, \quad i=1, \ldots, n
$$

where $r_{i} \in \mathbb{R}$ is the position and $u_{i} \in \mathbb{R}$ is the control input associated with the $i$ th vehicle. Here we have assumed that all vehicles are in a one-dimensional space for the simplicity of presentation. However, all results hereafter are still valid for the $m$-dimensional $(m>1)$ case by introduction of the Kronecker product.

\section{A. Distributed Consensus Tracking Under Fixed and Switching Network Topologies}

In this subsection, we design $u_{i}$ for (1) such that all followers track the virtual leader with local interaction in the absence of velocity measurements. We propose the distributed consensus tracking algorithm for (1) as

$$
u_{i}=-\alpha \sum_{j=0}^{n} a_{i j}\left(r_{i}-r_{j}\right)-\beta \operatorname{sgn}\left[\sum_{j=0}^{n} a_{i j}\left(r_{i}-r_{j}\right)\right]
$$

where $a_{i j}, i, j=1, \ldots, n$, is the $(i, j)$ th entry of the adjacency matrix $\mathcal{A}, a_{i 0}, i=1, \ldots, n$, is a positive constant if the virtual leader's position is available to follower $i$ and $a_{i 0}=0$ otherwise, $\alpha$ is a nonnegative constant, $\beta$ is a positive constant, and $\operatorname{sgn}(\cdot)$ is the signum function. We first consider the case of a fixed network topology.

Theorem 3.1: Suppose that the fixed undirected graph $\mathcal{G}$ is connected and at least one $a_{i 0}$ is nonzero (and hence positive). Using (2) for (1), if $\beta>\gamma_{\ell}$, then $r_{i}(t) \rightarrow r_{0}(t)$ in finite time. In particular, $r_{i}(t)=r_{0}(t)$ for any $t \geq \bar{t}$, where

$$
\bar{t}=\frac{\sqrt{\tilde{r}^{T}(0) M \tilde{r}(0)} \sqrt{\lambda_{\max }(M)}}{\left(\beta-\gamma_{\ell}\right) \lambda_{\min }(M)}
$$

where $\tilde{r}$ is the column stack vector of $\tilde{r}_{i}, i=1, \ldots, n$, with $\tilde{r}_{i}=r_{i}-r_{0}, M=\mathcal{L}+\operatorname{diag}\left(a_{10}, \ldots, a_{n 0}\right)$ with $\mathcal{L}$ being the Laplacian matrix, and $\lambda_{\min }(\cdot)$ and $\lambda_{\max }(\cdot)$ denote, respectively, the smallest and the largest eigenvalue of a symmetric matrix.

Proof: Noting that $\tilde{r}_{i}=r_{i}-r_{0}$, we can rewrite the closedloop system of (1) using (2) as

$$
\dot{\tilde{r}}_{i}=-\alpha \sum_{j=0}^{n} a_{i j}\left(\tilde{r}_{i}-\tilde{r}_{j}\right)-\beta \text { sgn }\left[\sum_{j=0}^{n} a_{i j}\left(\tilde{r}_{i}-\tilde{r}_{j}\right)\right]-\dot{r}_{0} .
$$


Equation (4) can be written in matrix form as

$$
\dot{\tilde{r}}=-\alpha M \tilde{r}-\beta \operatorname{sgn}(M \tilde{r})-1 \dot{r}_{0}
$$

where $\tilde{r}$ and $M$ are defined in (3), and $\operatorname{sgn}(\cdot)$ is defined componentwise. Because the fixed undirected graph $\mathcal{G}$ is connected and at least one $a_{i 0}$ is nonzero (and hence positive), $M$ is symmetric positive definite.

Consider the Lyapunov function candidate $V=(1 / 2) \tilde{r}^{T} M \tilde{r}$. The derivative of $V$ is

$$
\begin{aligned}
\dot{V} & =\tilde{r}^{T} M\left[-\alpha M \tilde{r}-\beta \operatorname{sgn}(M \tilde{r})-1 \dot{r}_{0}\right] \\
& \leq-\alpha \tilde{r}^{T} M^{2} \tilde{r}-\beta\|M \tilde{r}\|_{1}+\left|\dot{r}_{0}\right|\|M \tilde{r}\|_{1} \\
& \leq-\alpha \tilde{r}^{T} M^{2} \tilde{r}-\left(\beta-\gamma_{\ell}\right)\|M \tilde{r}\|_{1}
\end{aligned}
$$

where we have used the Hölder's inequality to obtain the first inequality and $\left|\dot{r}_{0}\right| \leq \gamma_{\ell}$ to obtain the second inequality. Note that $M^{2}$ is symmetric positive definite, $\alpha$ is nonnegative, and $\beta>\gamma_{\ell}$. Therefore, it follows that $\dot{V}$ is negative definite. It then follows from Theorem 3.1 in [19] that $\|\tilde{r}\| \rightarrow 0$ as $t \rightarrow \infty$.

We next show that $V$ will decrease to zero in finite time (i.e., $\tilde{r}_{i}(t) \rightarrow 0$ in finite time). Note that $V \leq(1 / 2) \lambda_{\max }(M)\|\tilde{r}\|_{2}^{2}$. It then follows from (5) that the derivative of $V$ satisfies

$$
\begin{aligned}
\dot{V} & \leq-\left(\beta-\gamma_{\ell}\right)\|M \tilde{r}\|_{2} \\
& =-\left(\beta-\gamma_{\ell}\right) \sqrt{\tilde{r}^{T} M^{2} \tilde{r}} \\
& \leq-\left(\beta-\gamma_{\ell}\right) \sqrt{\lambda_{\min }^{2}(M)\|\tilde{r}\|_{2}^{2}} \\
& =-\left(\beta-\gamma_{\ell}\right) \lambda_{\min }(M)\|\tilde{r}\|_{2} \\
& \leq-\left(\beta-\gamma_{\ell}\right) \frac{\sqrt{2} \lambda_{\min }(M)}{\sqrt{\lambda_{\max }(M)}} \sqrt{V} .
\end{aligned}
$$

After some manipulation, we can get that

$$
2 \sqrt{V(t)} \leq 2 \sqrt{V(0)}-\left(\beta-\gamma_{\ell}\right) \frac{\sqrt{2} \lambda_{\min }(M)}{\sqrt{\lambda_{\max }(M)}} t .
$$

Therefore, we have $V(t)=0$ when $t \geq \bar{t}$, where $\bar{t}$ is given by (3). This completes the proof.

Let $\overline{\mathcal{N}}_{i} \subseteq\{0,1, \ldots, n\}$ denote the neighbor set of follower $i$ in the team consisting of the $n$ followers and the virtual leader. We next consider the case of a switching network topology by assuming that $j \in \overline{\mathcal{N}}_{i}(t), i=1, \ldots, n, j=0, \ldots, n$, if $\mid r_{i}-$ $r_{j} \mid<R$ at time $t$ and $j \notin \overline{\mathcal{N}}_{i}(t)$ otherwise, where $R$ denotes the communication/sensing radius of the vehicles. In this case, we consider the distributed consensus tracking algorithm for (1) $u_{i}=-\alpha \sum_{j \in \overline{\mathcal{N}}_{i}(t)} b_{i j}\left(r_{i}-r_{j}\right)-\beta \operatorname{sgn}\left[\sum_{j \in \overline{\mathcal{N}}_{i}(t)} b_{i j}\left(r_{i}-r_{j}\right)\right]$

where $b_{i j}, i=1, \ldots, n, j=0, \ldots, n$, are positive constants, and $\alpha, \beta$, and $\operatorname{sgn}(\cdot)$ are defined as in (2).

Theorem 3.2: Suppose that the undirected graph $\mathcal{G}(t)$ is connected and the virtual leader is a neighbor of at least one follower (i.e., $0 \in \overrightarrow{\mathcal{N}}_{i}(t)$ for some $i$ ) at each time instant. Using (6) for (1), if $\beta>\gamma_{\ell}$, then $r_{i}(t) \rightarrow r_{0}(t)$ as $t \rightarrow \infty$.

Proof: Let $V_{i j}=(1 / 2) b_{i j}\left(r_{i}-r_{j}\right)^{2}, i, j=1, \ldots, n$, when $\left|r_{i}-r_{j}\right|<R$ and $V_{i j}=(1 / 2) b_{i j} R^{2}$ when $\left|r_{i}-r_{j}\right| \geq R$. Also let $V_{i 0}=(1 / 2) b_{i 0}\left(r_{i}-r_{0}\right)^{2}, i=1, \ldots, n$, when $\left|r_{i}-r_{0}\right|<R$ and $V_{i 0}=(1 / 2) b_{i 0} R^{2}$ when $\left|r_{i}-r_{0}\right| \geq R$. Consider the Lyapunov function candidate $V=(1 / 2) \sum_{i=1}^{n} \sum_{j=1}^{n} V_{i j}+$ $\sum_{i=1}^{n} V_{i 0}$. Note that $V$ is not smooth but is regular. We use differential inclusions [20], [21] and nonsmooth analysis [22], [23] to analyze the stability of (1) using (6). Therefore, the closed-loop system of (1) using (6) can be written as

$$
\begin{gathered}
\dot{r}_{i} \in^{a . e .}-K\left[\alpha \sum_{j \in \overline{\mathcal{N}}_{i}(t)} b_{i j}\left(r_{i}-r_{j}\right)\right. \\
\left.+\beta \operatorname{sgn}\left[\sum_{j \in \overline{\mathcal{N}}_{i}(t)} b_{i j}\left(r_{i}-r_{j}\right)\right]\right]
\end{gathered}
$$

where $K[\cdot]$ is the differential inclusion [21] and a.e. stands for "almost everywhere."

The generalized derivative of $V$ is given by

$$
\begin{aligned}
& V^{o}=\frac{1}{2} \sum_{i=1}^{n} \sum_{j=1}^{n} b_{i j}\left[\frac{\partial V_{i j}}{\partial r_{i}} \dot{r}_{i}+\frac{\partial V_{i j}}{\partial r_{j}} \dot{r}_{j}\right] \\
& +\sum_{i=1}^{n} b_{i 0}\left[\frac{\partial V_{i 0}}{\partial r_{i}} \dot{r}_{i}+\frac{\partial V_{i 0}}{\partial r_{0}} \dot{r}_{0}\right] \\
& =\frac{1}{2} \sum_{i=1}^{n} \sum_{j \in \overline{\mathcal{N}}_{i}(t), j \neq 0} b_{i j}\left[\left(r_{i}-r_{j}\right) \dot{r}_{i}+\left(r_{j}-r_{i}\right) \dot{r}_{j}\right] \\
& +\sum_{0 \in \overline{\mathcal{N}}_{i}(t)} b_{i 0}\left[\left(r_{i}-r_{0}\right) \dot{r}_{i}+\left(r_{0}-r_{i}\right) \dot{r}_{0}\right] \\
& =-\alpha \sum_{i=1}^{n}\left[\sum_{j \in \overline{\mathcal{N}}_{i}(t)} b_{i j}\left(r_{i}-r_{j}\right)\right]^{2} \\
& -\beta \sum_{i=1}^{n}\left|\sum_{j \in \overline{\mathcal{N}}_{i}(t)} b_{i j}\left(r_{i}-r_{j}\right)\right| \\
& +\sum_{0 \in \overline{\mathcal{N}}_{i}(t)} b_{i 0}\left(r_{0}-r_{i}\right) \dot{r}_{0} \\
& =-\alpha \tilde{r}^{T}[\hat{M}(t)]^{2} \tilde{r}-\beta\|\hat{M}(t) \tilde{r}\|_{1} \\
& +\dot{r}_{0} \sum_{0 \in \overline{\mathcal{N}}_{i}(t)} b_{i 0}\left(r_{0}-r_{i}\right) \\
& +\dot{r}_{0} \sum_{i=1}^{n} \sum_{j \in \mathcal{\mathcal { N }}_{i}(t), j \neq 0} b_{i j}\left(r_{i}-r_{j}\right) \\
& =-\alpha \tilde{r}^{T}[\hat{M}(t)]^{2} \tilde{r}-\beta\|\hat{M}(t) \tilde{r}\|_{1} \\
& +\dot{r}_{0} \sum_{i=1}^{n} \sum_{j \in \overline{\mathcal{N}}_{i}(t)} b_{i j}\left(r_{i}-r_{j}\right) \\
& \leq-\alpha \tilde{r}^{T}[\hat{M}(t)]^{2} \tilde{r}-\beta\|\hat{M}(t) \tilde{r}\|_{1}+\dot{r}_{0}\|\hat{M}(t) \tilde{r}\|_{1} \\
& \leq-\alpha \tilde{r}^{T}[\hat{M}(t)]^{2} \tilde{r}-\left(\beta-\gamma_{\ell}\right)\|\hat{M}(t) \tilde{r}\|_{1}
\end{aligned}
$$

where we have used the fact $\sum_{i=1}^{n} \sum_{j \in \overline{\mathcal{N}}_{i}(t), j \neq 0} b_{i j}\left(r_{i}-r_{j}\right)=$ 0 to derive (8) and $\left|\dot{r}_{0}\right| \leq \gamma_{\ell}$ to derive (9), $\tilde{r}$ is the column stack vector of $\tilde{r}_{i}, i=1, \ldots, n$, with $\tilde{r}_{i}=r_{i}-r_{0}$, and $\hat{M}(t)=$ $\left[\hat{m}_{i j}(t)\right] \in \mathbb{R}^{n \times n}$ is defined as

$$
\hat{m}_{i j}(t)= \begin{cases}-b_{i j}, & j \in \overline{\mathcal{N}}_{i}(t), j \neq i \\ 0, & j \notin \overline{\mathcal{N}}_{i}(t), j \neq i \\ \sum_{k \in \overline{\mathcal{N}}_{i}(t)} b_{i k}, & j=i\end{cases}
$$


Note that $\hat{M}(t)$ is symmetric positive definite at each time instant under the condition of the theorem. Because $\beta>\gamma_{\ell}$, it then follows that the generalized derivative of $V$ is negative definite under the condition of the theorem. It then follows from Theorem 3.1 in [19] that $\|\tilde{r}(t)\| \rightarrow 0$ as $t \rightarrow \infty$. Therefore, we can get that $r_{i}(t) \rightarrow r_{0}(t)$ as $t \rightarrow \infty$.

Remark 3.3: Under the condition of Theorem 3.2, distributed consensus tracking can be achieved in finite time under a switching network topology. However, in contrast to the result in Theorem 3.1, it is not easy to explicitly compute the bound of the time (i.e., $\bar{t}$ in Theorem 3.1) because the switching pattern of the network topology also plays an important role in determining the bound of the time.

\section{B. Distributed Swarm Tracking Under a Switching Network Topology}

In this subsection, we extend the distributed consensus tracking algorithm in Section III-A to achieve distributed swarm tracking. The objective here is to design $u_{i}$ for (1) such that all followers move cohesively with the virtual leader while avoiding intervehicle collision with local interaction in the absence of velocity measurements. Before moving on, we need to define potential functions which will be used in the distributed swarm tracking algorithms.

Definition 3.4: The potential function $V_{i j}$ is a differentiable, nonnegative function of $\left\|r_{i}-r_{j}\right\|^{1}$ satisfying the following conditions.

1) $V_{i j}$ achieves its unique minimum when $\left\|r_{i}-r_{j}\right\|$ is equal to its desired value $d_{i j}$.

2) $V_{i j} \rightarrow \infty$ if $\left\|r_{i}-r_{j}\right\| \rightarrow 0$.

3) $\left(\partial V_{i j} / \partial\left(\left\|r_{i}-r_{j}\right\|\right)\right)=0$ if $\left\|r_{i}-r_{j}\right\| \geq R$, where $R>$ $\max _{i, j} d_{i j}$ is a positive constant.

4) $V_{i i}=c, i=1, \ldots, n$, where $c$ is a positive constant.

Lemma 3.1: Let $V_{i j}$ be defined in Definition 3.4. The following equality holds:

$$
\frac{1}{2} \sum_{i=1}^{n} \sum_{j=1}^{n}\left(\frac{\partial V_{i j}}{\partial r_{i}} \dot{r}_{i}+\frac{\partial V_{i j}}{\partial r_{j}} \dot{r}_{j}\right)=\sum_{i=1}^{n} \sum_{j=1}^{n} \frac{\partial V_{i j}}{\partial r_{i}} \dot{r}_{i} .
$$

Proof: Note that

$$
\begin{aligned}
& \frac{1}{2} \sum_{i=1}^{n} \sum_{j=1}^{n}\left(\frac{\partial V_{i j}}{\partial r_{i}} \dot{r}_{i}+\frac{\partial V_{i j}}{\partial r_{j}} \dot{r}_{j}\right) \\
& =\frac{1}{2} \sum_{i=1}^{n} \sum_{j=1}^{n} \frac{\partial V_{i j}}{\partial r_{i}} \dot{r}_{i}-\frac{1}{2} \sum_{i=1}^{n} \sum_{j=1}^{n} \frac{\partial V_{i j}}{\partial r_{i}} \dot{r}_{j} \\
& =\frac{1}{2} \sum_{i=1}^{n} \sum_{j=1}^{n} \frac{\partial V_{i j}}{\partial r_{i}} \dot{r}_{i}-\frac{1}{2} \sum_{j=1}^{n} \sum_{i=1}^{n} \frac{\partial V_{j i}}{\partial r_{j}} \dot{r}_{i} \\
& =\frac{1}{2} \sum_{i=1}^{n} \sum_{j=1}^{n} \frac{\partial V_{i j}}{\partial r_{i}} \dot{r}_{i}+\frac{1}{2} \sum_{j=1}^{n} \sum_{i=1}^{n} \frac{\partial V_{i j}}{\partial r_{i}} \dot{r}_{i} \\
& \quad=\sum_{i=1}^{n} \sum_{j=1}^{n} \frac{\partial V_{i j}}{\partial r_{i}} \dot{r}_{i},
\end{aligned}
$$

where we have used the fact that $\partial V_{i j} / \partial r_{i}=-\partial V_{i j} / \partial r_{j}$ from Definition 3.4. Therefore, the lemma holds.

\footnotetext{
${ }^{1}$ In this definition, $r_{i}$ can be $m$-dimensional.
}

We propose the distributed swarm tracking algorithm for (1) as

$$
u_{i}=-\alpha \sum_{j \in \overline{\mathcal{N}}_{i}(t)} \frac{\partial V_{i j}}{\partial r_{i}}-\beta \operatorname{sgn}\left(\sum_{j \in \overline{\mathcal{N}}_{i}(t)} \frac{\partial V_{i j}}{\partial r_{i}}\right)
$$

where $\alpha, \beta$, and $\overline{\mathcal{N}}_{i}(t)$ are defined as in Section III-A, and $V_{i j}$ is defined in Definition 3.4.

Theorem 3.5: Suppose that the undirected graph $\mathcal{G}(t)$ is connected and the virtual leader is a neighbor of at least one follower (i.e., $0 \in \overline{\mathcal{N}}_{i}(t)$ for some $i$ ) at each time instant. Using (11) for (1), if $\beta>\gamma_{\ell}$, the followers will stay close to the virtual leader and the intervehicle collision is avoided.

Proof: Consider the Lyapunov function candidate

$$
V=\frac{1}{2} \sum_{i=1}^{n} \sum_{j=1}^{n} V_{i j}+\sum_{i=1}^{n} V_{i 0} .
$$

Note that $V$ is continuously differentiable with respect to $r_{i}$ and $r_{j}$. It follows that

$$
\begin{aligned}
& \dot{V}=\frac{1}{2} \sum_{i=1}^{n} \sum_{j=1}^{n}\left(\frac{\partial V_{i j}}{\partial r_{i}} \dot{r}_{i}+\frac{\partial V_{i j}}{\partial r_{j}} \dot{r}_{j}\right) \\
& +\sum_{i=1}^{n}\left(\frac{\partial V_{i 0}}{\partial r_{i}} \dot{r}_{i}+\frac{\partial V_{i 0}}{\partial r_{0}} \dot{r}_{0}\right) \\
& =\sum_{i=1}^{n} \sum_{j=1}^{n} \frac{\partial V_{i j}}{\partial r_{i}} \dot{r}_{i}+\sum_{i=1}^{n}\left(\frac{\partial V_{i 0}}{\partial r_{i}} \dot{r}_{i}+\frac{\partial V_{i 0}}{\partial r_{0}} \dot{r}_{0}\right) \\
& =\sum_{i=1}^{n} \sum_{j=1}^{n} \frac{\partial V_{i j}}{\partial r_{i}}\left[-\alpha \sum_{j=0}^{n} \frac{\partial V_{i j}}{\partial r_{i}}-\beta \operatorname{sgn}\left(\sum_{j=0}^{n} \frac{\partial V_{i j}}{\partial r_{i}}\right)\right] \\
& +\sum_{i=1}^{n} \frac{\partial V_{i 0}}{\partial r_{i}}\left[-\alpha \sum_{j=0}^{n} \frac{\partial V_{i j}}{\partial r_{i}}-\beta \operatorname{sgn}\left(\sum_{j=0}^{n} \frac{\partial V_{i j}}{\partial r_{i}}\right)\right] \\
& +\sum_{i=1}^{n} \frac{\partial V_{i 0}}{\partial r_{0}} \dot{r}_{0} \\
& =-\alpha \sum_{i=1}^{n}\left(\sum_{j=0}^{n} \frac{\partial V_{i j}}{\partial r_{i}}\right)^{2}-\beta \sum_{i=1}^{n}\left|\sum_{j=0}^{n} \frac{\partial V_{i j}}{\partial r_{i}}\right| \\
& +\sum_{i=1}^{n} \frac{\partial V_{i 0}}{\partial r_{0}} \dot{r}_{0} \\
& =-\alpha \sum_{i=1}^{n}\left(\sum_{j=0}^{n} \frac{\partial V_{i j}}{\partial r_{i}}\right)^{2}-\beta \sum_{i=1}^{n}\left|\sum_{j=0}^{n} \frac{\partial V_{i j}}{\partial r_{i}}\right| \\
& +\sum_{i=1}^{n} \frac{\partial V_{i 0}}{\partial r_{0}} \dot{r}_{0}+\sum_{i=1}^{n} \sum_{j=1}^{n} \frac{\partial V_{i j}}{\partial r_{i}} \dot{r}_{0} \\
& \leq-\alpha \sum_{i=1}^{n}\left(\sum_{j=0}^{n} \frac{\partial V_{i j}}{\partial r_{i}}\right)^{2}-\beta \sum_{i=1}^{n}\left|\sum_{j=0}^{n} \frac{\partial V_{i j}}{\partial r_{i}}\right| \\
& +\left|\dot{r}_{0}\right| \sum_{i=1}^{n}\left|\sum_{j=0}^{n} \frac{\partial V_{i j}}{\partial r_{i}}\right|
\end{aligned}
$$

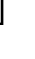

$$
\begin{aligned}
& \text {. }
\end{aligned}
$$

(n)




\section{Distributed COORDINATED TRACKING FOR SECOND-ORDER DYNAMICS}

In this section, we study distributed coordinated tracking for second-order dynamics. Suppose that there exists a virtual leader, labeled as vehicle 0 , with a (time-varying) position $r_{0}$ and velocity $v_{0}$. We consider four different cases.

\section{A. Distributed Consensus Tracking With a Varying Virtual Leader's Velocity}

Consider followers with second-order dynamics given by

$$
\dot{r}_{i}=v_{i}, \quad \dot{v}_{i}=u_{i}, \quad i=1, \ldots, n
$$

where $r_{i} \in \mathbb{R}$ and $v_{i} \in \mathbb{R}$ are, respectively, the position and velocity of follower $i$, and $u_{i} \in \mathbb{R}$ is the control input. We assume that $\left|\dot{v}_{0}\right| \leq \varphi_{\ell}$, where $\varphi_{\ell}$ is a positive constant. Again we only consider the case when all vehicles are in a one-dimensional space. All results hereafter are still valid for the $m$-dimensional $(m>1)$ case by introduction of the Kronecker product.

In this subsection, we assume that the virtual leader has a varying velocity (i.e., $v_{0}$ is time-varying). The objective here is to design $u_{i}$ for (14) such that all followers track the virtual leader with local interaction in the absence of acceleration measurements. We propose the distributed consensus tracking algorithm for (14) as

$$
\begin{aligned}
u_{i}=-\sum_{j=0}^{n} a_{i j}\left[\left(r_{i}-r_{j}\right)+\alpha\left(v_{i}-v_{j}\right)\right] \\
-\beta \operatorname{sgn}\left\{\sum_{j=0}^{n} a_{i j}\left[\gamma\left(r_{i}-r_{j}\right)+\left(v_{i}-v_{j}\right)\right\}\right.
\end{aligned}
$$

where $a_{i j}, i=1, \ldots, n, j=0, \ldots, n$, is defined as in (2), and $\alpha, \beta$, and $\gamma$ are positive constants. We first consider the case of a fixed network topology. Before moving on, we need the following lemma.

Lemma 4.1: Suppose that the fixed undirected graph $\mathcal{G}$ is connected and at least one $a_{i 0}$ is nonzero (and hence positive). Let

$$
\begin{aligned}
& P=\left[\begin{array}{cc}
(1 / 2) M^{2} & (\gamma / 2) M \\
(\gamma / 2) M & (1 / 2) M
\end{array}\right] \\
& Q=\left[\begin{array}{cc}
\gamma M^{2} & (\alpha \gamma / 2) M^{2} \\
(\alpha \gamma / 2) M^{2} & \alpha M^{2}-\gamma M
\end{array}\right]
\end{aligned}
$$

where $\gamma$ and $\alpha$ are positive constants and $M=\mathcal{L}+$ $\operatorname{diag}\left(a_{10}, \ldots, a_{n 0}\right)$. If $\gamma$ satisfies

$$
0<\gamma<\min \left\{\sqrt{\lambda_{\min }(M)}, \frac{4 \alpha \lambda_{\min }(M)}{4+\alpha^{2} \lambda_{\min }(M)}\right\}
$$

then both $P$ and $Q$ are symmetric positive definite.

Proof: When the fixed undirected graph $\mathcal{G}$ is connected and at least one $a_{i 0}$ is nonzero (and hence positive), $M$ is symmetric positive definite. It follows that $M$ can be diagonalized as $M=$ $\Gamma^{-1} \Lambda \Gamma$, where $\Lambda=\operatorname{diag}\left\{\lambda_{1}, \ldots, \lambda_{n}\right\}$ with $\lambda_{i}$ being the $i$ th eigenvalue of $M$. It then follows that $P$ can be written as

$$
P=\left[\begin{array}{cc}
\Gamma^{-1} & \mathbf{0}_{n \times n} \\
\mathbf{0}_{n \times n} & \Gamma^{-1}
\end{array}\right] \underbrace{\left[\begin{array}{cc}
\frac{1}{2} \Lambda^{2} & \frac{\gamma}{2} \Lambda \\
\frac{\gamma}{2} \Lambda & \frac{1}{2} \Lambda
\end{array}\right]}_{F}\left[\begin{array}{cc}
\Gamma & \mathbf{0}_{n \times n} \\
\mathbf{0}_{n \times n} & \Gamma
\end{array}\right]
$$

where $0_{n \times n}$ is the $n \times n$ zero matrix. Let $\mu$ be an eigenvalue of $F$. Because $\Lambda$ is a diagonal matrix, it follows from (17) that $\mu$ satisfies $\left(\mu-(1 / 2) \lambda_{i}^{2}\right)\left(\mu-(1 / 2) \lambda_{i}\right)-\left(\gamma^{2} / 4\right) \lambda_{i}^{2}=0$, which can be simplified as

$$
\mu^{2}-\frac{1}{2}\left(\lambda_{i}^{2}+\lambda_{i}\right) \mu+\frac{1}{4}\left(\lambda_{i}^{3}-\gamma^{2} \lambda_{i}^{2}\right)=0 .
$$

Because $F$ is symmetric, the roots of (18) are real. Therefore, all roots of (18) are positive if and only if $(1 / 2)\left(\lambda_{i}^{2}+\lambda_{i}\right)>0$ and $1 / 4\left(\lambda_{i}^{3}-\gamma^{2} \lambda_{i}^{2}\right)>0$. Because $\lambda_{i}>0$, it follows that $1 / 2\left(\lambda_{i}^{2}+\right.$ $\left.\lambda_{i}\right)>0$. When $\gamma^{2}<\lambda_{i}$, it follows that $1 / 4\left(\lambda_{i}^{3}-\gamma^{2} \lambda_{i}^{2}\right)>0$. It thus follows that when $\gamma^{2}<\lambda_{i}$, the roots of (18) are positive. Noting that $P$ has the same eigenvalues as $F$, we can get that $P$ is positive definite if $0<\gamma<\sqrt{\lambda_{\min }(M)}$.

By following a similar analysis, we can get that $Q$ is positive definite if $0<\gamma<4 \alpha \lambda_{\min }(M) / 4+\alpha^{2} \lambda_{\min }(M)$. Combining the above arguments proves the lemma.

Theorem 4.1: Suppose that the fixed undirected graph $\mathcal{G}$ is connected and at least one $a_{i 0}$ is nonzero (and hence positive). Using (15) for (14), if $\beta>\varphi_{\ell}$ and $\gamma$ satisfies (16), then $r_{i}(t) \rightarrow$ $r_{0}(t)$ and $v_{i}(t) \rightarrow v_{0}(t)$ globally exponentially as $t \rightarrow \infty$. In particular, it follows that

$$
\left\|\left[\begin{array}{ll}
\tilde{r}^{T}(t) & \tilde{v}^{T}(t)
\end{array}\right]^{T}\right\|_{2} \leq \kappa_{1} e^{-\kappa_{2} t}
$$

where $\tilde{r}$ and $\tilde{v}$ are, respectively, the column stack vectors of $\tilde{r}_{i}$ and $\tilde{v}_{i}, i=1, \ldots, n$, with $\tilde{r}_{i}=r_{i}-r_{0}$ and $\tilde{v}_{i}=v_{i}-v_{0}, P$ and $Q$ are defined in Lemma 4.1, $\kappa_{1}=\sqrt{\left[\begin{array}{lll}\tilde{r}^{T}(0) & \left.\tilde{v}^{T}(0)\right] P\left[\tilde{r}^{T}(0)\right. & \tilde{v}^{T}(0)\end{array}\right]^{T} / \lambda_{\min }(P)}$, and $\kappa_{2}=\lambda_{\min }(Q) / 2 \lambda_{\max }(P)$.

Proof: Noting that $\tilde{r}_{i}=r_{i}-r_{0}$ and $\tilde{v}_{i}=v_{i}-v_{0}$, we rewrite the closed-loop system of (14) using (15) as

$$
\begin{aligned}
\dot{\tilde{r}}_{i}= & \tilde{v}_{i} \\
\dot{\tilde{v}}_{i}= & -\sum_{j=0}^{n} a_{i j}\left[\left(\tilde{r}_{i}-\tilde{r}_{j}\right)+\alpha\left(\tilde{v}_{i}-\tilde{v}_{j}\right)\right] \\
& -\beta \operatorname{sgn}\left\{\sum_{j=0}^{n} a_{i j}\left[\gamma\left(\tilde{r}_{i}-\tilde{r}_{j}\right)+\left(\tilde{v}_{i}-\tilde{v}_{j}\right)\right]\right\}-\dot{v}_{0} .
\end{aligned}
$$

Equation (20) can be written in matrix form as

$$
\begin{aligned}
& \dot{\tilde{r}}=\tilde{v} \\
& \dot{\tilde{v}}=-M \tilde{r}-\alpha M \tilde{v}-\beta \operatorname{sgn}[M(\gamma \tilde{r}+\tilde{v})]-\mathbf{1} \dot{v}_{0}
\end{aligned}
$$

where $\tilde{r}$ and $\tilde{v}$ are defined in (19) and $M=\mathcal{L}+$ $\operatorname{diag}\left(a_{10}, \ldots, a_{n 0}\right)$.

Consider the Lyapunov function candidate

$$
\begin{aligned}
V & =\left[\begin{array}{ll}
\tilde{r}^{T} & \tilde{v}^{T}
\end{array}\right] P\left[\begin{array}{l}
\tilde{r} \\
\tilde{v}
\end{array}\right] \\
& =\frac{1}{2} \tilde{r}^{T} M^{2} \tilde{r}+\frac{1}{2} \tilde{v}^{T} M \tilde{v}+\gamma \tilde{r}^{T} M \tilde{v} .
\end{aligned}
$$

Note that according to Lemma 4.1, $P$ is symmetric positive definite when $\gamma$ satisfies (16). The derivative of $V$ is

$$
\begin{aligned}
\dot{V} & =\tilde{r}^{T} M^{2} \tilde{v}+\tilde{v}^{T} M \dot{\tilde{v}}+\gamma \tilde{v}^{T} M \tilde{v}+\gamma \tilde{r}^{T} M \dot{\tilde{v}} \\
& =-\left[\begin{array}{ll}
\tilde{r}^{T} & \tilde{v}^{T}
\end{array}\right] Q\left[\begin{array}{l}
\tilde{r} \\
\tilde{v}
\end{array}\right]
\end{aligned}
$$




$$
\begin{aligned}
& -\left(\gamma \tilde{r}^{T}+\tilde{v}^{T}\right) M\left\{\beta \operatorname{sgn}[M(\gamma \tilde{r}+\tilde{v})]+1 \dot{v}_{0}\right\} \\
\leq & -\left[\begin{array}{ll}
\tilde{r}^{T} & \tilde{v}^{T}
\end{array}\right] Q\left[\begin{array}{l}
\tilde{r} \\
\tilde{v}
\end{array}\right]-\left(\beta-\varphi_{\ell}\right)\|M(\gamma \tilde{r}+\tilde{v})\|_{1}
\end{aligned}
$$

where the last inequality follows from the fact that $\left|\dot{v}_{0}\right| \leq \varphi_{\ell}$. Note that according to Lemma 4.1, $Q$ is symmetric positive definite when $\gamma$ satisfies (16). Also note that $\beta>\varphi_{\ell}$. It follows that $\dot{V}$ is negative definite. Therefore, it follows from Theorem 3.1 in [19] that $\tilde{r}(t) \rightarrow \mathbf{0}_{n}$ and $\tilde{v}(t) \rightarrow \mathbf{0}_{n}$ as $t \rightarrow \infty$, where $\mathbf{0}_{n}$ is the $n \times 1$ zero vector. Equivalently, it follows that $r_{i}(t) \rightarrow r_{0}(t)$ and $v_{i}(t) \rightarrow v_{0}(t)$ as $t \rightarrow \infty$.

We next show that distributed consensus tracking is achieved at least globally exponentially. Note that $V \leq \lambda_{\max }(P)\left\|\left[\begin{array}{cc}\tilde{r}^{T} & \tilde{v}^{T}\end{array}\right]^{T}\right\|_{2}^{2}$. It then follows from (22) that

$$
\begin{aligned}
\dot{V} & \leq-\left[\begin{array}{ll}
\tilde{r}^{T} & \tilde{v}^{T}
\end{array}\right] Q\left[\begin{array}{c}
\tilde{r} \\
\tilde{v}
\end{array}\right] \\
& \leq-\lambda_{\min }(Q)\left\|\left[\begin{array}{ll}
\tilde{r}^{T} & \tilde{v}^{T}
\end{array}\right]^{T}\right\|_{2}^{2} \leq-\frac{\lambda_{\min }(Q)}{\lambda_{\max }(P)} V .
\end{aligned}
$$

Therefore, we can get that $V(t) \leq V(0) e^{-\lambda_{\min }(Q) / \lambda_{\max }(P) t}$. Note also that $V \geq \lambda_{\min }(P)\left\|\left[\begin{array}{ll}\tilde{r}^{T} & \tilde{v}^{T}\end{array}\right]^{T}\right\|_{2}^{2}$. After some manipulation, we can get (19).

Remark 4.2: In the proof of Theorem 4.1, the Lyapunov function is chosen as (21). Here $P$ can also be chosen as

$$
P=\left[\begin{array}{ll}
(1 / 2) M & (\gamma / 2) M \\
(\gamma / 2) M & (1 / 2) M
\end{array}\right]
$$

and the derivative of $V$ also satisfies (22) with

$$
Q=\left[\begin{array}{cc}
\gamma M^{2} & (\alpha \gamma / 2) M^{2}+\left(M^{2}-\gamma M / 2\right) \\
(\alpha \gamma / 2) M^{2}+\left(M^{2}-\gamma M / 2\right) & \alpha M^{2}-\gamma M
\end{array}\right] .
$$

By following a similar analysis to that of Lemma 4.1, we can show that there always exist positive $\alpha$ and $\gamma$ such that both $P$ and $Q$ are symmetric positive definite and derive proper conditions for $\alpha$ and $\gamma$. In particular, one special choice for $\alpha$ and $\gamma$ is $\alpha \gamma=1$ and $\gamma<\left(4 \lambda_{\min }(M) / 4 \lambda_{\min }(M)+1\right)$.

We next consider the case of a switching network topology. We assume that the network topology switches according to the same model as described right before (6). In this case, we propose the distributed consensus tracking algorithm for (14) as

$$
\begin{aligned}
u_{i}= & -\sum_{j \in \overline{\mathcal{N}}_{i}(t)} b_{i j}\left[\left(r_{i}-r_{j}\right)+\alpha\left(v_{i}-v_{j}\right)\right] \\
- & \beta \sum_{j \in \overline{\mathcal{N}}_{i}(t)} b_{i j}\left(\operatorname{sgn}\left\{\sum_{k \in \overline{\mathcal{N}}_{i}(t)} b_{i k}\left[\gamma\left(r_{i}-r_{k}\right)+\left(v_{i}-v_{k}\right)\right]\right\}\right. \\
& \left.-\operatorname{sgn}\left\{\sum_{k \in \overline{\mathcal{N}}_{j}(t)} b_{j k}\left[\gamma\left(r_{j}-r_{k}\right)+\left(v_{j}-v_{k}\right)\right]\right\}\right)
\end{aligned}
$$

where $\overline{\mathcal{N}}_{i}(t)$ is defined as in Section III-A, $b_{i j}, i=1, \ldots, n$, $j=0, \ldots, n$, are positive constants, and $\alpha, \beta$, and $\gamma$ are positive constants. ${ }^{2}$ Before moving on, we need the following lemma.

Lemma 4.2: Suppose that the undirected graph $\mathcal{G}(t)$ is connected and the virtual leader is a neighbor of at least one follower

$$
\begin{aligned}
& { }^{2} \text { Because the virtual leader has no neighbor, we let } \\
& \qquad \operatorname{sgn}\left\{\sum_{k \in \overline{\mathcal{N}}_{0}(t)} b_{0 k}\left[\gamma\left(r_{0}-r_{k}\right)+\left(v_{0}-v_{k}\right)\right]\right\}=0 .
\end{aligned}
$$

(i.e., $0 \in \overline{\mathcal{N}}_{i}(t)$ for some $i$ ) at each time instant. Let $\hat{M}(t)$ be defined as in (10). Let

$$
\hat{P}(t)=\left[\begin{array}{cc}
(1 / 2) \hat{M}(t) & (\gamma / 2) I_{n} \\
(\gamma / 2) I_{n} & (1 / 2) I_{n}
\end{array}\right]
$$

and

$$
\hat{Q}(t)=\left[\begin{array}{cc}
\gamma \hat{M}(t) & (\alpha \gamma / 2) \hat{M}(t) \\
(\alpha \gamma / 2) \hat{M}(t) & \alpha \hat{M}(t)-\gamma I_{n}
\end{array}\right]
$$

where $\gamma$ and $\alpha$ are positive constants. If $\gamma$ satisfies

$$
0<\gamma<\min _{t}\left\{\sqrt{\lambda_{\min }(\hat{M}(t))}, \frac{4 \alpha \lambda_{\min }(\hat{M}(t))}{4+\alpha^{2} \lambda_{\min }(\hat{M}(t))}\right\}
$$

then both $\hat{P}(t)$ and $\hat{Q}(t)$ are symmetric positive definite at each time instant.

Proof: The proof is similar to that of Lemma 4.1 and is therefore omitted here.

Theorem 4.3: Suppose that the undirected graph $\mathcal{G}(t)$ is connected and the virtual leader is a neighbor of at least one follower (i.e., $0 \in \overline{\mathcal{N}}_{i}(t)$ for some $i$ ) at each time instant. Using (23) for (14), if $\beta>\varphi_{\ell}$ and (24) is satisfied, then $r_{i}(t) \rightarrow r_{0}(t)$ and $v_{i}(t) \rightarrow v_{0}(t)$ as $t \rightarrow \infty$.

Proof: Let $V_{i j}$ and $V_{i 0}$ be defined as in the proof of Theorem 3.2. Consider the Lyapunov function candidate $V=$ $(1 / 2) \sum_{i=1}^{n} \sum_{j=1}^{n} V_{i j}+\sum_{i=1}^{n} V_{i 0}+(1 / 2) \tilde{v}^{T} \tilde{v}+\gamma \tilde{r}^{T} \tilde{v}$, where $\tilde{r}=\left[\tilde{r}_{1}, \ldots, \tilde{r}_{n}\right]^{T}$ with $\tilde{r}_{i}=r_{i}-r_{0}$ and $\tilde{v}=\left[\tilde{v}_{1}, \ldots, \tilde{v}_{n}\right]^{T}$ with $\tilde{v}_{i}=v_{i}-v_{0}$. Note that $V$ can be written as

$$
\begin{aligned}
V= & {\left[\begin{array}{ll}
\tilde{r}^{T} & \tilde{v}^{T}
\end{array}\right] \hat{P}(t)\left[\begin{array}{l}
\tilde{r} \\
\tilde{v}
\end{array}\right]+\frac{1}{4} \sum_{i=1}^{n} \sum_{j \notin \overline{\mathcal{N}}_{i}(t), j \neq 0} b_{i j} R^{2} } \\
& +\frac{1}{2} \sum_{0 \in \overline{\mathcal{N}}_{i}(t)} b_{i 0} R^{2} .
\end{aligned}
$$

Note also that according to Lemma 4.2, $\hat{P}(t)$ is symmetric positive definite when (24) is satisfied. By following a similar line to the proof of Theorem 4.1 and using nonsmooth analysis, we can obtain that the generalized derivative of $V$ is negative definite under the condition of the theorem. Therefore, it follows from Theorem 3.1 in [19] that $r_{i}(t) \rightarrow r_{0}(t)$ and $v_{i}(t) \rightarrow v_{0}(t)$ as $t \rightarrow \infty$.

Remark 4.4: It can be noted that (23) requires the availability of the information from both the neighbors (i.e., one-hop neighbors) and the neighbors' neighbors (i.e., two-hop neighbors). However, accurate measurements of the two-hop neighbors' information are not necessary because only the signs (i.e., "+" or "-") are required in (23). In fact, (23) can be easily implemented in real systems in the sense that follower $i, i=1, \ldots, n$, shares both its own state (i.e., position and velocity) and the sign of $\sum_{j \in \overline{\mathcal{N}}_{i}(t)} b_{i j}\left[\gamma\left(r_{i}-r_{j}\right)+\left(v_{i}-v_{j}\right)\right]$ with its neighbors. Note that follower $i$ also has to compute $\sum_{j \in \overline{\mathcal{N}}_{i}(t)} b_{i j}\left(r_{i}-r_{j}\right)$ and $\sum_{j \in \overline{\mathcal{N}}_{i}(t)} b_{i j}\left(v_{i}-v_{j}\right)$ in (23) [correspondingly, $\sum_{j=0}^{n} a_{i j}\left(r_{i}-\right.$ $\left.r_{j}\right)$ and $\sum_{j=0}^{n} a_{i j}\left(v_{i}-v_{j}\right)$ in (15)] in order to derive the corresponding control input for itself.

Remark 4.5: Under the condition of Theorem 4.3, the distributed consensus tracking algorithm (23) guarantees at least global exponential tracking under a switching network topology. However, in contrast to the result in Theorem 4.1, it might not be easy to explicitly compute the decay rate (i.e., $\kappa_{2}$ in Theorem 4.3) because the switching pattern of the network 
topology will play an important role in determining the decay rate.

Remark 4.6: Similar to the analysis in Remark 4.2, in Lyapunov function (25), we can choose

It then follows that

$$
\hat{P}(t)=\left[\begin{array}{ll}
(1 / 2) I_{n} & (\gamma / 2) I_{n} \\
(\gamma / 2) I_{n} & (1 / 2) I_{n}
\end{array}\right]
$$

$\hat{Q}(t)=\left[\begin{array}{cc}\gamma \hat{M}(t) & (\alpha \gamma / 2) \hat{M}(t)+\left(\hat{M}(t)-\gamma I_{n}\right) / 2 \\ (\alpha \gamma / 2) \hat{M}(t)+\left(\hat{M}(t)-\gamma I_{n} / 2\right) & \alpha \hat{M}(t)-\gamma I_{n}\end{array}\right]$.

We can show that there always exist positive $\alpha$ and $\gamma$ such that both $\hat{P}(t)$ and $\hat{Q}(t)$ are symmetric positive definite and derive proper conditions for $\alpha$ and $\gamma$. In particular, one special choice for $\alpha$ and $\gamma$ is $\alpha \gamma=1$ and $\left.\left.\gamma<\min _{t} 4 \lambda_{\min }[\hat{M}(t)] /\left(4 \lambda_{\min }[\hat{M}(t)]+1\right)\right]\right)$.

Remark 4.7: In Theorems 3.2 and 4.3, it is assumed that the undirected graph $\mathcal{G}(t)$ is connected and the virtual leader is a neighbor of at least one follower at each time instant. However, this poses an obvious constraint in real applications because the connectivity requirement is not necessarily always satisfied. Next, we propose an adaptive connectivity maintenance mechanism in which the adjacency matrix with entries $b_{i j}$ in (6) and (23) is redefined as follows:

1) $b_{i j}$ is a function of $\left\|r_{i}-r_{j}\right\|$.

2) When $\left\|r_{i}(0)-r_{j}(0)\right\| \geq R, b_{i j}(t)=1$ if $\left\|r_{i}(t)-r_{j}(t)\right\|<$ $R$ and $b_{i j}(t)=0$ otherwise.

3) When $\left\|r_{i}(0)-r_{j}(0)\right\|<R, b_{i j}(t)$ is defined satisfying: 1) $b_{i j}(0)>0$;2) $b_{i j}(t)$ is nondecreasing; 3$) b_{i j}(t)$ is differentiable (or differentiable almost everywhere); 4) $b_{i j}(t)$ goes to infinity if $\left\|r_{i}(t)-r_{j}(t)\right\|$ goes to $R$.

The motivation here is to maintain the initially existing connectivity patterns. That is, if two followers are neighbors of each other (correspondingly, the virtual leader is a neighbor of a follower) at $t=0$, the two followers are guaranteed to be neighbors of each other (correspondingly, the virtual leader is guaranteed to be a neighbor of this follower) at $t>0$. However, if two followers are not neighbors of each other (correspondingly, the virtual leader is not a neighbor of a follower) at $t=0$, the two followers are not necessarily guaranteed to be neighbors of each other (correspondingly, the virtual leader is not necessarily guaranteed to be a neighbor of this follower) at $t>0$.

Using the proposed adaptive adjacency matrix, the consensus tracking algorithm for (1) can be chosen as

$$
\begin{aligned}
& u_{i}=-\alpha \sum_{j \in \overline{\mathcal{N}}_{i}(t)} b_{i j}(t)\left(r_{i}-r_{j}\right)-\beta \sum_{j \in \overline{\mathcal{N}}_{i}(t)} b_{i j}(t) \\
& \left\{\operatorname{sgn}\left[\sum_{k \in \overline{\mathcal{N}}_{i}(t)} b_{i k}(t)\left(r_{i}-r_{k}\right)\right]-\operatorname{sgn}\left[\sum_{k \in \overline{\mathcal{N}}_{j}(t)} b_{j k}(t)\left(r_{j}-r_{k}\right)\right]\right\}
\end{aligned}
$$

with the Lyapunov function chosen as $V=(1 / 2) \tilde{r}^{T} \tilde{r}$ while the consensus tracking algorithm for (14) can be chosen as (23) with the Lyapunov function chosen as $V=\left[\begin{array}{ll}\tilde{r}^{T} & \tilde{v}^{T}\end{array}\right] \hat{P}(t)\left[\begin{array}{c}\tilde{r} \\ \tilde{v}\end{array}\right]$ with $\hat{P}(t)$ chosen as in Remark 4.6. According to the definition of $\hat{M}(t)$, for $0 \leq t_{1}<t_{2}, x^{T}\left[\hat{M}\left(t_{1}\right)-\hat{M}\left(t_{2}\right)\right] x \leq 0$ for all vectors $x \in \mathbb{R}^{n}$ under the connectivity maintenance mechanism. Let $y \in \mathbb{R}^{n}$ be the right eigenvector of $\hat{M}\left(t_{2}\right)$ associated with the eigenvalue $\lambda_{\min }\left[\hat{M}\left(t_{2}\right)\right]$, i.e.,
$\hat{M}\left(t_{2}\right) y=\lambda_{\min }\left[\hat{M}\left(t_{2}\right)\right] y$. It follows that $y^{T} \lambda_{\min }\left[\hat{M}\left(t_{2}\right)\right] y=$ $y^{T} \hat{M}\left(t_{2}\right) y \geq y^{T} \hat{M}\left(t_{1}\right) y \geq y^{T} \lambda_{\min }\left[\hat{M}\left(t_{1}\right)\right] y$. Because $y^{T} y \neq 0$, it follows that $\lambda_{\min }\left[\hat{M}\left(t_{2}\right)\right] \geq \lambda_{\min }\left[\hat{M}\left(t_{1}\right)\right]$. This implies that $\lambda_{\min }[\hat{M}(t)]$ is nondecreasing with respect to time. Therefore, there always exist $\alpha$ and $\gamma$ satisfying the conditions in Remark 4.6 because $\lambda_{\min }[\hat{M}(t)]$ is nondecreasing under the connectivity maintenance mechanism. When the control gains are chosen properly (i.e., $\alpha>0$ and $\beta>\gamma_{\ell}$ for first-order kinematics and $\alpha$ and $\gamma$ satisfies Remark 4.6 and $\beta>\varphi_{\ell}$ for second-order dynamics), it can be shown that distributed consensus tracking can be guaranteed for both first-order kinematics and second-order dynamics if the undirected graph $\mathcal{G}(t)$ is initially connected and the virtual leader is initially a neighbor of at least one follower (i.e., at $t=0$ ). The proof follows a similar analysis to that of the corresponding algorithm in the absence of connectivity maintenance mechanism except that the initially existing connectivity patterns can be maintained because otherwise $\dot{V} \rightarrow-\infty$ as $\left\|r_{i}(t)-r_{j}(t)\right\| \rightarrow R$ by noting that $b_{i j}(t)\left|r_{i}(t)-r_{j}(t)\right| \rightarrow \infty$ as $\left\|r_{i}(t)-r_{j}(t)\right\| \rightarrow R$, $\dot{V}=-\alpha \tilde{r} \hat{M}(t) \tilde{r}-\left(\beta-\gamma_{\ell}\right)\|\hat{M}(t) \tilde{r}\|$ for first-order kinematics, and $\dot{V}=-\left[\begin{array}{ll}\tilde{r}^{T} & \tilde{v}^{T}\end{array}\right] \hat{Q}(t)\left[\begin{array}{l}\tilde{r} \\ \tilde{v}\end{array}\right]$ for second-order dynamics, where $\hat{Q}(t)$ is defined in Remark 4.6.

\section{B. Distributed Consensus Tracking With a Constant Virtual Leader's Velocity}

In this subsection, we assume that the virtual leader has a constant velocity (i.e., $v_{0}$ is constant). We propose the distributed consensus tracking algorithm for (14) as

$$
u_{i}=-\sum_{j=0}^{n} a_{i j}\left(r_{i}-r_{j}\right)-\beta \operatorname{sgn}\left[\sum_{j=0}^{n} a_{i j}\left(v_{i}-v_{j}\right)\right]
$$

where $a_{i j}$ is defined as in (15) and $\beta$ is a positive constant. We first consider a fixed network topology.

Theorem 4.8: Suppose that the fixed undirected graph $\mathcal{G}$ is connected and at least one $a_{i 0}$ is nonzero (and hence positive). Using (27) for (14), $r_{i}(t) \rightarrow r_{0}(t)$ and $v_{i}(t) \rightarrow v_{0}$ as $t \rightarrow \infty$.

Proof: Letting $\tilde{r}_{i}=r_{i}-r_{0}$ and $\tilde{v}_{i}=v_{i}-v_{0}$, we can rewrite the closed-loop system of (14) using (27) as

$$
\begin{aligned}
& \dot{\tilde{r}}_{i}=\tilde{v}_{i} \\
& \dot{\tilde{v}}_{i}=-\sum_{j=0}^{n} a_{i j}\left(\tilde{r}_{i}-\tilde{r}_{j}\right)-\beta \operatorname{sgn}\left[\sum_{j=0}^{n} a_{i j}\left(\tilde{v}_{i}-\tilde{v}_{j}\right)\right] .
\end{aligned}
$$

Equation (28) can be written in matrix form as

$$
\dot{\tilde{r}}=\tilde{v}, \quad \dot{\tilde{v}}=-M \tilde{r}-\beta \operatorname{sgn}(M \tilde{v})
$$

where $\tilde{r}$ and $\tilde{v}$ are, respectively, the column stack vectors of $\tilde{r}_{i}$ and $\tilde{v}_{i}, i=1, \ldots, n$, and $M=\mathcal{L}+\operatorname{diag}\left(a_{10}, \ldots, a_{n 0}\right)$.

Consider the Lyapunov function candidate $V=(1 / 2) \tilde{r}^{T} M^{2} \tilde{r}+(1 / 2) \tilde{v}^{T} M \tilde{v}$. The derivative of $V$ is given by

$$
\begin{aligned}
\dot{V} & =\tilde{r}^{T} M^{2} \tilde{v}+\tilde{v}^{T} M \dot{\tilde{v}} \\
& =\tilde{r}^{T} M^{2} \tilde{v}+\tilde{v}^{T} M[-M \tilde{r}-\beta \operatorname{sgn}(M \tilde{v})] \\
& =-\beta\|M \tilde{v}\|_{1} .
\end{aligned}
$$


Because $M$ is symmetric positive definite, it follows that $\dot{V}$ is negative semidefinite. Note that $\dot{V} \equiv 0$ implies that $\tilde{v} \equiv \mathbf{0}_{n}$, which in turn implies that $\tilde{r} \equiv \mathbf{0}_{n}$ from (29). By using Theorem 3.2 in [19], it follows that $\tilde{r}(t) \rightarrow \mathbf{0}_{n}$ and $\tilde{v}(t) \rightarrow \mathbf{0}_{n}$ as $t \rightarrow \infty$. Equivalently, it follows that $r_{i}(t) \rightarrow r_{0}(t)$ and $v_{i}(t) \rightarrow v_{0}(t)$ as $t \rightarrow \infty$.

Remark 4.9: In contrast to (15) and (23), which require both accurate position and velocity measurements, (27) does not necessarily require accurate velocity measurements because the velocity measurements are only used to calculate the sign (i.e., "+" or " -"). Therefore, (27) is more robust to measurement inaccuracy.

\section{Distributed Swarm Tracking With a Constant Virtual Leader's Velocity}

In this subsection, we study distributed swarm tracking under switching network topologies when the velocity of the virtual leader is constant. We again assume that the network topology switches according to the model described right before (6). We propose the distributed swarm tracking algorithm for (14) as

$$
\begin{aligned}
& u_{i}=-\sum_{j \in \overline{\mathcal{N}}_{i}(t)} \frac{\partial V_{i j}}{\partial r_{i}} \\
&-\beta \sum_{j \in \overline{\mathcal{N}}_{i}(t)} b_{i j}\left\{\operatorname{sgn}\left[\sum_{k \in \overline{\mathcal{N}}_{i}(t)} b_{i k}\left(v_{i}-v_{k}\right)\right]\right. \\
&\left.-\operatorname{sgn}\left[\sum_{k \in \overline{\mathcal{N}}_{j}(t)} b_{j k}\left(v_{j}-v_{k}\right)\right]\right\}
\end{aligned}
$$

where $V_{i j}$ is the potential function defined in Definition 3.4, $\overline{\mathcal{N}}_{i}(t)$ is defined as in Section III-A, $\beta$ is a positive constant, and $b_{i j}, b_{i k}$, and $b_{j k}$ are defined as in (23). Note that (30) requires both the one-hop and two-hop neighbors' information.

Theorem 4.10: Suppose that the undirected graph $\mathcal{G}(t)$ is connected and the virtual leader is a neighbor of at least one follower (i.e., $0 \in \overline{\mathcal{N}}_{i}(t)$ for some $i$ ) at each time instant. Using (30) for (14), the velocity differences of all followers and the virtual leader will ultimately converge to zero (i.e., the intervehicle distance will be maintained), $\lim _{t \rightarrow \infty} \sum_{j \in \overline{\mathcal{N}}_{i}(t)}\left(\partial V_{i j} / \partial r_{i}\right)=0$, $i=1, \ldots, n$, and the intervehicle collision is avoided.

Proof: Letting $\tilde{r}_{i}=r_{i}-r_{0}$ and $\tilde{v}_{i}=v_{i}-v_{0}$, it follows that (30) can be written as

$$
\begin{aligned}
u_{i}=- & \sum_{j \in \overline{\mathcal{N}}_{i}(t)} \frac{\partial V_{i j}}{\partial \tilde{r}_{i}} \\
-\beta \sum_{j \in \overline{\mathcal{N}}_{i}(t)} b_{i j}\{\operatorname{sgn} & {\left[\sum_{k \in \overline{\mathcal{N}}_{i}(t)} b_{i k}\left(\tilde{v}_{i}-\tilde{v}_{k}\right)\right] } \\
& \left.-\operatorname{sgn}\left[\sum_{k \in \overline{\mathcal{N}}_{j}(t)} b_{j k}\left(\tilde{v}_{j}-\tilde{v}_{k}\right)\right]\right\} .
\end{aligned}
$$

Consider the Lyapunov function candidate

$$
V=\frac{1}{2} \sum_{i=1}^{n} \sum_{j=1}^{n} V_{i j}+\sum_{i=1}^{n} V_{i 0}+\frac{1}{2} \tilde{v}^{T} \tilde{v}
$$

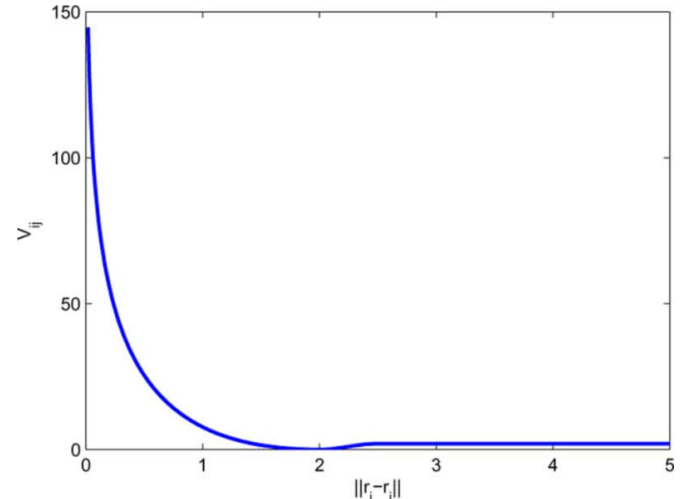

(a)

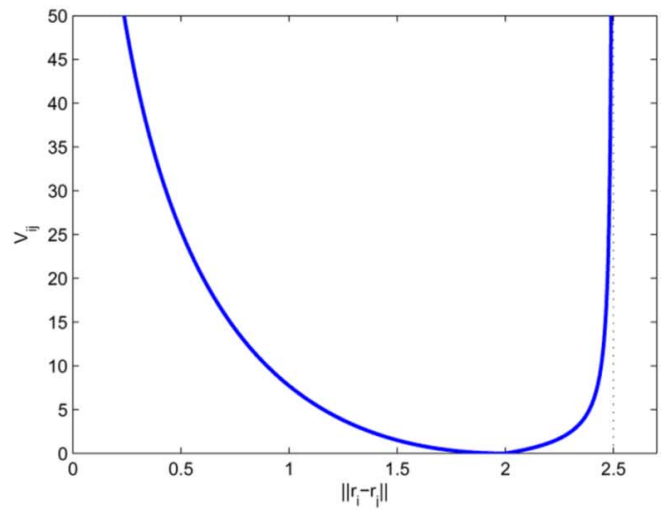

(b)

Fig. 1. Potential functions $V_{i j}^{1}$ and $V_{i j}^{2}$ with $R=2.5$ and $d_{i j}=2$. (a) Potential function $V_{i j}^{1}$. (b) Potential function $V_{i j}^{2}$.

where $\tilde{v}$ is a column stack vector of $\tilde{v}_{i}$. Taking derivative of $V$ gives that

$$
\begin{aligned}
\dot{V}= & \frac{1}{2} \sum_{i=1}^{n} \sum_{j=1}^{n}\left(\frac{\partial V_{i j}}{\partial \tilde{r}_{i}} \dot{\tilde{r}}_{i}+\frac{\partial V_{i j}}{\partial \tilde{r}_{j}} \dot{\tilde{r}}_{j}\right) \\
& +\sum_{i=1}^{n}\left(\frac{\partial V_{i 0}}{\partial \tilde{r}_{i}} \dot{\tilde{r}}_{i}+\frac{\partial V_{i 0}}{\partial \tilde{r}_{0}} \dot{\tilde{r}}_{0}\right)+\tilde{v}^{T} \dot{\tilde{v}} \\
= & \sum_{i=1}^{n} \sum_{j=1}^{n} \frac{\partial V_{i j}}{\partial \tilde{r}_{i}} \dot{\tilde{r}}_{i}+\sum_{i=1}^{n} \frac{\partial V_{i 0}}{\partial \tilde{r}_{i}} \dot{\tilde{r}}_{i}-\sum_{i=1}^{n} \tilde{v}_{i} \sum_{j=0}^{n} \frac{\partial V_{i j}}{\partial \tilde{r}_{i}} \\
& -\beta \tilde{v}^{T} \hat{M}(t) \operatorname{sgn}[\hat{M}(t) \tilde{v}] \\
= & -\beta\|\hat{M}(t) \tilde{v}\|_{1}
\end{aligned}
$$

where $\hat{M}(t)$ is defined in (10), (31) is derived by using Lemma 3.1 and the fact that $\dot{\tilde{r}}_{0}=0$, and (32) is derived by using the fact that $\hat{M}(t)$ is symmetric. By following a similar analysis to that in the proof of Theorem 4.8, it follows from Theorem 3.2 in [19] that $v_{i}(t) \rightarrow v_{0}$ and $\sum_{j=0}^{n}\left(\partial V_{i j} / \partial r_{i}\right) \rightarrow 0$ as $t \rightarrow \infty$, which in turn proves the theorem.

\section{Distributed Swarm Tracking With a Varying Virtual Leader's Velocity}

In this subsection, we assume that the virtual leader's velocity is varying (i.e., the virtual leader's acceleration is, in general, 


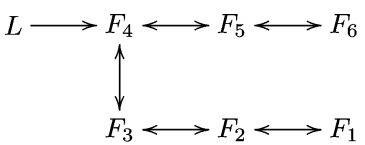

(a)

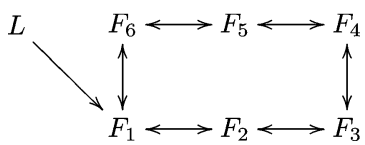

(b)

Fig. 2. Network topology for a group of six followers with a virtual leader. Here $L$ denotes the virtual leader while $F_{i}, i=1, \ldots, 6$, denote the followers.

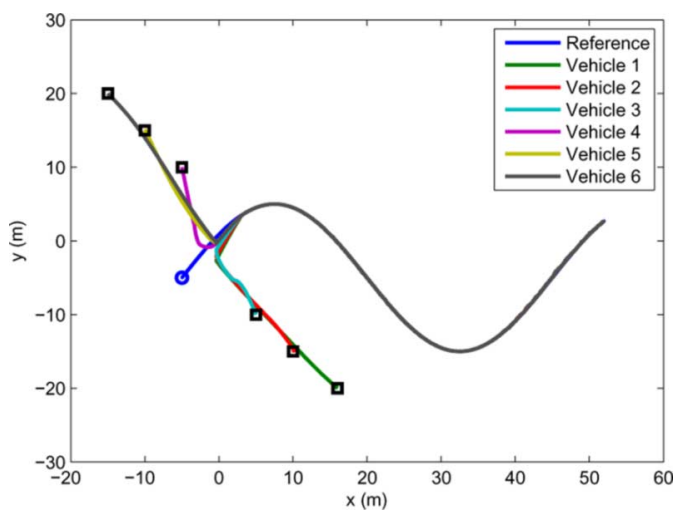

Fig. 3. Trajectories of the followers and the virtual leader using (2) in 2-D. The circle denotes the starting position of the virtual leader while the squares denote the starting positions of the follwers.

nonzero.). We propose the following distributed swarm tracking algorithm with a distributed estimator for (14) as

$$
\begin{aligned}
u_{i}= & -\gamma \operatorname{sgn}\left\{\sum_{j \in \overline{\mathcal{N}}_{i}(t)} b_{i j}\left[\hat{v}_{i 0}-\hat{v}_{j 0}\right]\right\}-\sum_{j \in \overline{\mathcal{N}}_{i}(t)} \frac{\partial V_{i j}}{\partial r_{i}} \\
-\beta \sum_{j \in \overline{\mathcal{N}}_{i}(t)} b_{i j}\{\operatorname{sgn} & {\left[\sum_{k \in \overline{\mathcal{N}}_{i}(t)} b_{i k}\left(v_{i}-v_{k}\right)\right] } \\
& \left.-\operatorname{sgn}\left[\sum_{k \in \overline{\mathcal{N}}_{j}(t)} b_{j k}\left(v_{j}-v_{k}\right)\right]\right\}
\end{aligned}
$$

where $\gamma$ and $\beta$ are positive constants, $V_{i j}, \overline{\mathcal{N}}_{i}(t), b_{i j}, b_{i k}$, and $b_{k j}$ are defined in (30), and

$$
\dot{\hat{v}}_{i 0}=-\gamma \mathrm{sgn}\left\{\sum_{j \in \overline{\mathcal{N}}_{i}(t)} b_{i j}\left[\hat{v}_{i 0}-\hat{v}_{j 0}\right]\right\}, \quad i=1, \ldots, n
$$

with $\hat{v}_{i 0}$ being the $i$ th vehicle's estimate of the virtual leader's velocity and $\hat{v}_{00}=v_{0}$. Here (34) is a distributed estimator motivated by the results in Section III-A.

Theorem 4.11: Suppose that the undirected graph $\mathcal{G}(t)$ is connected and the virtual leader is a neighbor of at least one follower (i.e., $0 \in \overline{\mathcal{N}}_{i}(t)$ for some $i$ ) at each time instant. Using (33) for (14), if $\gamma>\varphi_{\ell}$, the velocity differences of all followers and the virtual leader will ultimately converge to zero (i.e., the intervehicle distance will be maintained), $\lim _{t \rightarrow \infty} \sum_{j \in \overline{\mathcal{N}}_{i}(t)} \partial V_{i j} / \partial r_{i}=0, i=1, \ldots, n$, and the intervehicle collision is avoided.

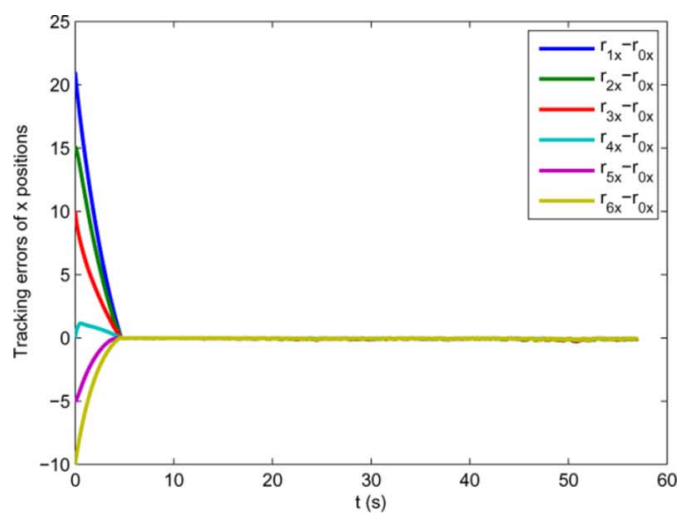

(a)

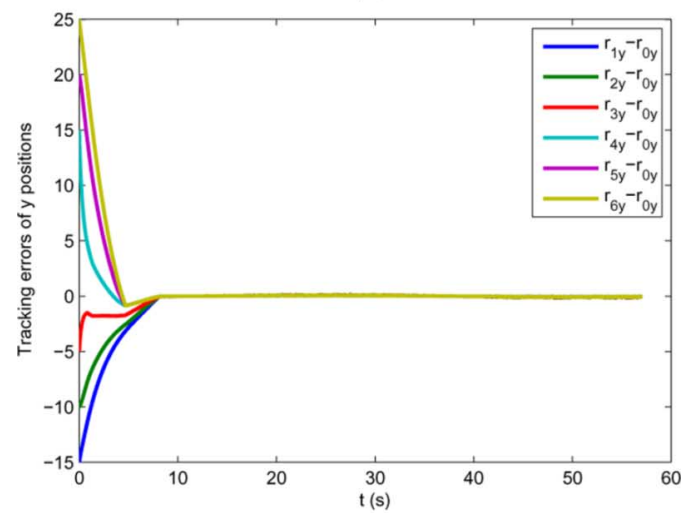

(b)

Fig. 4. Position tracking errors using (2) in 2-D.

Proof: Letting $\tilde{r}_{i} \triangleq r_{i}-r_{0}$ and $\tilde{v}_{i} \triangleq v_{i}-v_{0}$, we can rewrite the closed-loop system of (14) using (33) as

$$
\begin{aligned}
\dot{\tilde{r}}_{i}= & \tilde{v}_{i} \\
\dot{\tilde{v}}_{i}= & -\gamma \operatorname{sgn}\left\{\sum_{j \in \overline{\mathcal{N}}_{i}(t)} b_{i j}\left[\hat{v}_{i 0}-\hat{v}_{j 0}\right]\right\}-\sum_{j \in \overline{\mathcal{N}}_{i}(t)} \frac{\partial V_{i j}}{\partial \tilde{r}_{i}} \\
& -\beta \sum_{j \in \overline{\mathcal{N}}_{i}(t)} b_{i j}\left\{\operatorname{sgn}\left[\sum_{k \in \overline{\mathcal{N}}_{i}(t)} b_{i k}\left(\tilde{v}_{i}-\tilde{v}_{k}\right)\right]\right. \\
& \left.-\operatorname{sgn}\left[\sum_{k \in \overline{\mathcal{N}}_{j}(t)} b_{j k}\left(\tilde{v}_{j}-\tilde{v}_{k}\right)\right]\right\}-\dot{v}_{0} .
\end{aligned}
$$

For (34), it follows from Theorem 3.2 that there exists positive $\bar{t}$ such that $\hat{v}_{i 0}(t) \equiv v_{0}(t)$ for any $t \geq \bar{t}$. Note that $\dot{\hat{v}}_{i 0}$ in (34) is a switching signal, which is different from $\dot{v}_{0}(t)$ at each time instant. However, for $\bar{t} \leq t_{1} \leq t_{2}$, we have that $\int_{t_{1}}^{t_{2}} \dot{\hat{v}}_{i 0}(t) d t=\int_{t_{1}}^{t_{2}} \dot{v}_{0}(t) d t$ by noting that $\hat{v}_{i 0}(t) \equiv v_{0}(t)$ for any $t \geq \bar{t}$. Therefore, $r_{i}$ and $v_{i}$ will remain unchanged when $-\gamma_{\text {sgn }}\left\{\sum_{j \in \overline{\mathcal{N}}_{i}(t)} b_{i j}\left[\hat{v}_{i 0}-\hat{v}_{j 0}\right]\right\}$ (equivalently, $\dot{\hat{v}}_{i 0}$ ) in (35) is replaced with $\dot{v}_{0}$ for $t \geq \bar{t}$. For $0 \leq t \leq \bar{t}$, by choosing the same Lyapunov function candidate as in the proof of Theorem 4.10, we can get that

$$
\begin{aligned}
\dot{V} & \leq-\beta\|\hat{M}(t) \tilde{v}\|_{1}+\left(\gamma+\varphi_{\ell}\right)\|\tilde{v}\|_{1} \\
& \leq\left(\gamma+\varphi_{\ell}\right) \sqrt{n}\|\tilde{v}\|_{2} \\
& \leq\left(\gamma+\varphi_{\ell}\right) \sqrt{n} \sqrt{2 V}
\end{aligned}
$$




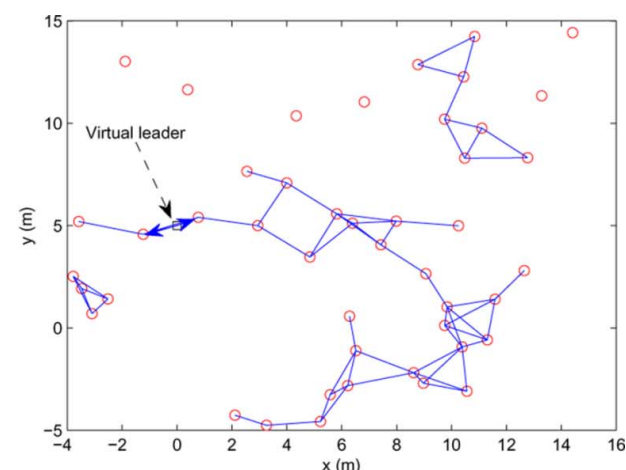

(a)

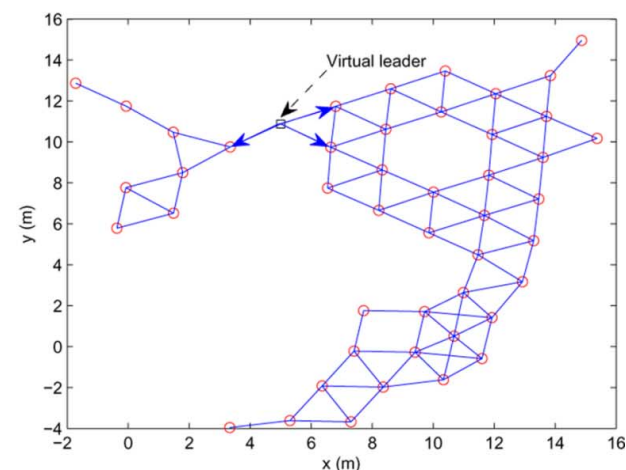

(b)

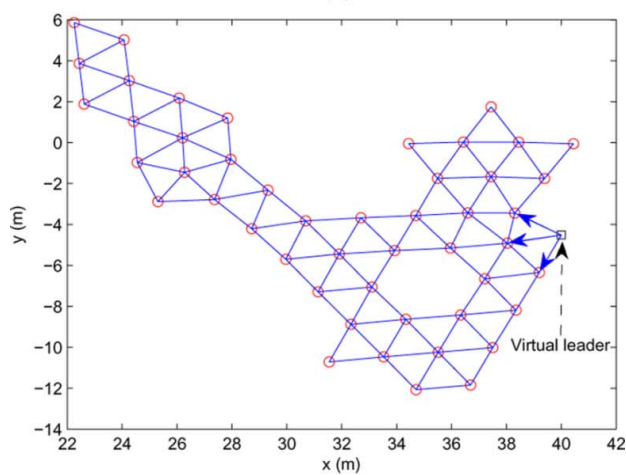

(c)

Fig. 5. Distributed swarm tracking for 48 followers with a virtual leader using (11) in 2-D. The circles denote the positions of the followers while the square denotes the position of the virtual leader. An undirected edge connecting two followers means that the two followers are neighbors of each other while a directed edge from the virtual leader to a follower means that the virtual leader is a neighbor of the follower.

which implies that $V(t) \leq\left(\sqrt{V(0)}+\left(\gamma+\varphi_{\ell}\right) \sqrt{n} / \sqrt{2} \bar{t}\right)^{2}$ for $0 \leq t \leq \bar{t}$. That is, $V(t)$ is bounded for $0 \leq t \leq \bar{t}$. For $t>\bar{t}$, by replacing $-\gamma_{\mathrm{sgn}}\left\{\sum_{j \in \overline{\mathcal{N}}_{i}(t)} b_{i j}\left[\hat{v}_{i 0}-\hat{v}_{j 0}\right]\right\}$ (equivalently, $\dot{\hat{v}}_{i 0}$ ) in (35) with $\dot{v}_{0}$ and choosing the same Lyapunov function candidate as in the proof of Theorem 4.10, we can get that $\dot{V} \leq-\beta\|\hat{M}(t) \tilde{v}\|_{1}$. It follows from a similar analysis to that in the proof of Theorem 4.10 and Theorem 3.2 in [19] that $\left|v_{i}(t)-v_{0}(t)\right| \rightarrow 0$ and $\sum_{j \in \overline{\mathcal{N}}_{i}(t)} \partial V_{i j} / \partial r_{i} \rightarrow 0$ as $t \rightarrow \infty$. This completes the proof.

Remark 4.12: Note that (30) and (33) require the availability of both the one-hop and two-hop neighbors' information. The availability of the leader's information (i.e., the position, velocity, and acceleration) to all followers is not required in (30)

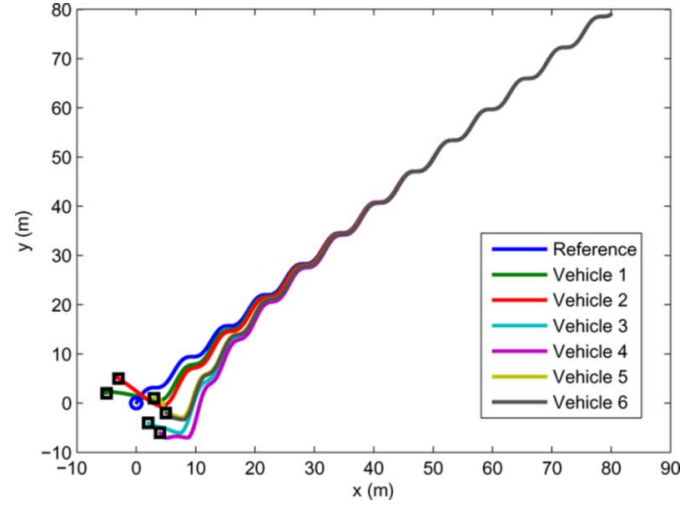

Fig. 6. Trajectories of the followers and the virtual leader using (15) in 2-D. The circle denotes the starting position of the virtual leader while the squares denote the starting positions of the followers.

due to the fact that $v_{0}$ is constant and in (33) due to the introduction of the distributed estimator. In addition, (30) does not require accurate velocity measurements of the leader and the followers while (33) does not require accurate velocity measurements of the followers because the velocity measurements are only used to calculate the signs (i.e., "+" or "-"). Therefore, (30) and (33) are robust to velocity measurement inaccuracy.

Remark 4.13: In Theorems 3.5, 4.10, and 4.11, it is assumed that the undirected graph $\mathcal{G}(t)$ is connected and the virtual leader is a neighbor of at least one follower at each time instant. However, this poses an obvious constraint in real applications because the connectivity requirement is not necessarily always satisfied. In the following, a mild connectivity requirement is proposed for distributed swarm tracking by adopting a connectivity maintenance mechanism in which the potential function in Definition 3.4 is redefined as follows.

1) When $\left\|r_{i}-r_{j}\right\| \geq R$ at the initial time (i.e., $t=0$ ), $V_{i j}$ is defined as in Definition 3.4.

2) When $\left\|r_{i}-r_{j}\right\|<R$ at the initial time (i.e., $t=0$ ), $V_{i j}$ is defined satisfying conditions 1), 2), and 4) in Definition 3.4 and condition 3) in Definition 3.4 is replaced with the condition that $V_{i j} \rightarrow \infty$ as $\left\|r_{i}-r_{j}\right\| \rightarrow R$. The motivation here is also to maintain the initially existing connectivity patterns as in Remark 4.7.

Using the potential function defined above, distributed swarm tracking can be guaranteed for both first-order kinematics (cf. Theorem 3.5) and second-order dynamics (cf. Theorems 4.10 and 4.11) if the undirected graph $\mathcal{G}(t)$ is initially connected (i.e., $t=0$ ), the virtual leader is initially a neighbor of at least one follower, and the other conditions for the control gains aresatisfied. The proof follows directly from those of Theorems 3.5, 4.10, and 4.11 except that a pair of followers who are neighbors of each other initially will always be the neighbors of each other (correspondingly, if the virtual leader is initially a neighbor of a follower, the virtual leader will always be a neighbor of this follower) because otherwise the potential function will go to infinity. This contradicts the fact that $\dot{V} \leq 0$ as shown in the proofs of Theorems 3.5 and 4.10 and the facts that $V(t)$ is bounded for $0 \leq t<\bar{t}$ and $\dot{V} \leq 0$ for $t \geq \bar{t}$ as shown in the proof of Theorem 4.11. Note that the connectivity maintenance strategy in [24] requires that the number of edges be always nondecreasing. That is, if a pair of followers are neighbors of each other (respectively, the virtual leader is a neighbor of a follower) at some time instant 


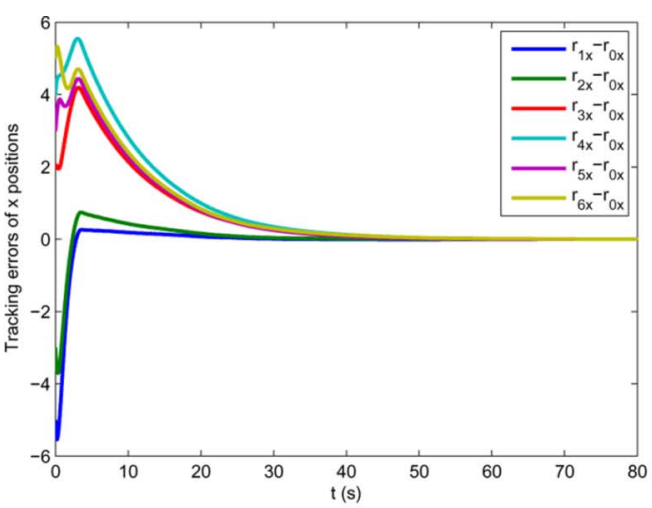

(a)

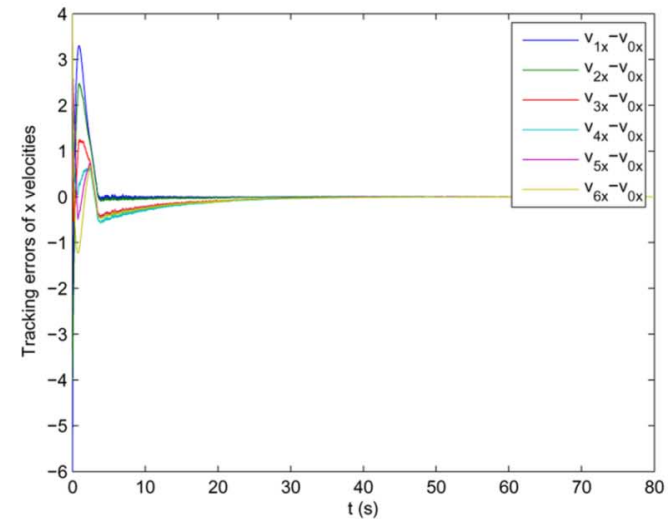

(c)

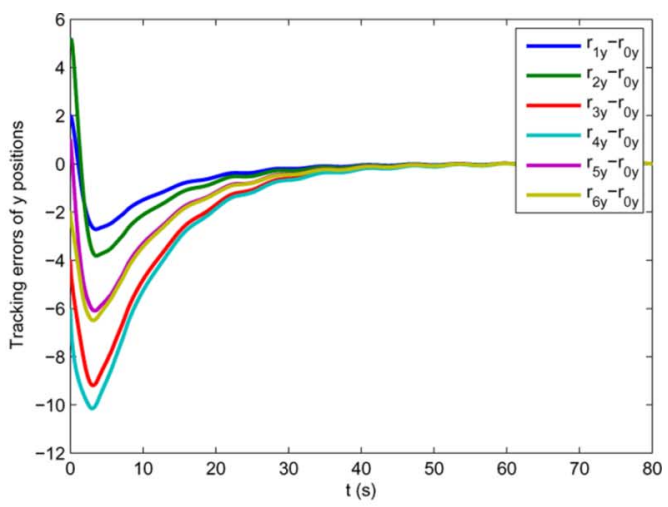

(b)

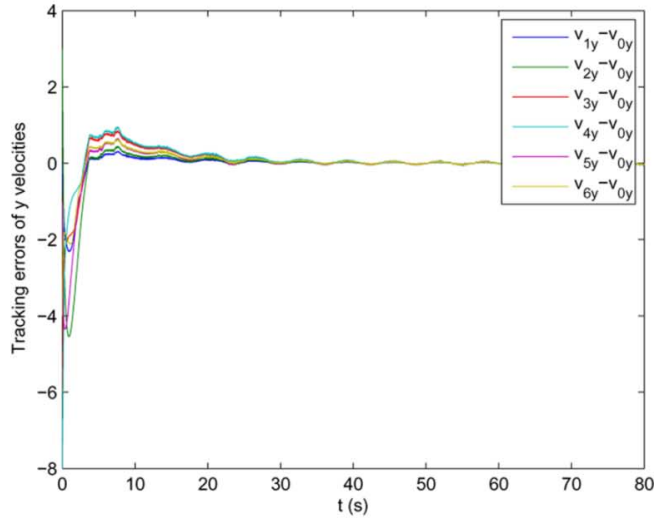

(d)

Fig. 7. Position and velocity tracking errors using (15) in 2-D. (a) $x$ position. (b) $y$ position. (c) $x$ velocity. (d) $y$ velocity.

$T,{ }^{3}$ then the pair of followers are always neighbors of each other (respectively, the virtual leader is always a neighbor of this follower) at any time $t>T$. This requirement might not be applicable in reality, especially in large-scale systems where the size of the vehicles cannot be ignored because the group of vehicles will become very compact with the increasing number of edges. Meanwhile, the computation burden will increase significantly as well. In contrast, the connectivity maintenance mechanism with the corresponding potential function proposed in Remark 4.13 takes these practical issues into consideration. In addition, hysteresis is introduced to the connectivity maintenance strategy in [24] to avoid the singularity of the Lyapunov function. However, the hysteresis is not required in the potential function proposed in Remark 4.13.

To illustrate the connectivity maintenance mechanism as proposed in Remark 4.13, we compare two different potential functions $V_{i j}^{1}$ and $V_{i j}^{2}$ whose derivatives satisfy, respectively

$$
\begin{aligned}
& \frac{\partial V_{i j}^{1}}{\partial r_{i}}= \\
& \begin{cases}0, & \left\|r_{i}-r_{j}\right\|>R \\
\frac{2 \pi\left(r_{i}-r_{j}\right) \sin \left[2 \pi\left(\left\|r_{i}-r_{j}\right\|-d_{i j}\right)\right]}{\left\|r_{i}-r_{j}\right\|}, & d_{i j}<\left\|r_{i}-r_{j}\right\| \leq R \\
\frac{20\left(r_{i}-r_{j}\right)}{\left\|r_{i}-r_{j}\right\|} \frac{\left\|r_{i}-r_{j}\right\|-d_{i j}}{\left\|r_{i}-r_{j}\right\|}, & \left\|r_{i}-r_{j}\right\| \leq d_{i j}\end{cases}
\end{aligned}
$$

and

$$
\frac{\partial V_{i j}^{2}}{\partial r_{i}}= \begin{cases}\frac{r_{i}-r_{j}}{\left\|r_{i}-r_{j}\right\|} \frac{\left\|r_{i}-r_{j}\right\|-d_{i j}}{\left(\left\|r_{i}-r_{j}\right\|-R\right)^{2}}, & d_{i j}<\left\|r_{i}-r_{j}\right\|<R \\ 20 \frac{r_{i}-r_{j}}{\left\|r_{i}-r_{j}\right\|} \frac{\left\|r_{i}-r_{j}\right\|-d_{i j}}{\left\|r_{i}-r_{j}\right\|}, & \left\|r_{i}-r_{j}\right\| \leq d_{i j}\end{cases}
$$

${ }^{3}$ Equivalently, a pair of followers are within the communication range of each other (respectively, the virtual leader is within the communication range of a follower). where $R=2.5$ and $d_{i j}=2$. Fig. 1 shows the plot of the potential functions $V_{i j}^{1}$ and $V_{i j}^{2}{ }^{4}$ It can be seen from Fig. 1(b) that $V_{i j}^{2}$ approaches infinity as the distance $\left\|r_{i}-r_{j}\right\|$ approaches $R$. However, $V_{i j}^{1}$ does not have the property [cf. Fig. 1(a)]. In particular, $V_{i j}^{1}$ satisfies condition 3 ) in Definition 3.4 as shown in Fig. 1(a). In addition, both $V_{i j}^{1}$ and $V_{i j}^{2}$ satisfy conditions 1 ), 2), and 4) in Definition 3.4. According to Remark 4.13, we can choose the potential function as $V_{i j}^{2}$ when $\left\|r_{i}(0)-r_{j}(0)\right\|<R$ and $V_{i j}^{1}$ otherwise.

Remark 4.14: From the proofs of all theorems, it can be seen that the condition that $\mathcal{G}(t)$ is undirected connected and the leader is a neighbor of at least one follower in each theorem can be relaxed to be that $\mathcal{G}(t)$ is undirected and the leader has directed paths to all followers.

\section{Simulation}

In this section, we present several simulation examples to validate some theoretical results in the previous sections. We consider a group of six followers with a virtual leader. We let $r_{i} \triangleq\left[r_{i x}, r_{i y}\right]^{T}$ and $v_{i} \triangleq\left[v_{i x}, v_{i y}\right]^{T}$, where $r_{i x}$ and $r_{i y}$ denote, respectively, the $x$ and $y$ positions of agent $i$ while $v_{i x}$ and $v_{i y}$ denote, respectively, the $x$ and $y$ velocities of agent $i$. We also let $a_{i j}=1$ if vehicle $j$ is a neighbor of vehicle $i$, where $j=0,1, \ldots, 6$ and $i=1, \ldots, 6$, and $a_{i j}=0$ otherwise.

In the case of first-order kinematics, the network topology is chosen as in Fig. 2(a). It can be noted that the undirected graph $\mathcal{G}$ for all followers 1 to 6 is connected and the virtual leader is a neighbor of follower 4. Using (2) in 2-D, we choose

${ }^{4}$ Note that neither $V_{i j}^{1}$ nor $V_{i j}^{2}$ is unique because for positive constant $C$, $V_{i j}^{1}+C$ and $V_{i j}^{2}+C$ are also potential functions satisfying, respectively, (36) and (37). We only plot one possible choice for them. 


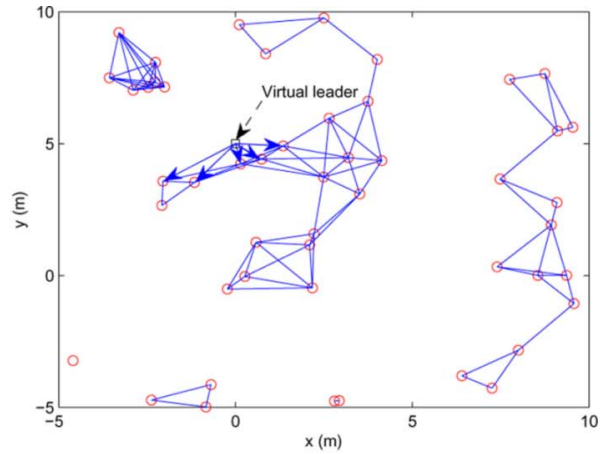

(a)

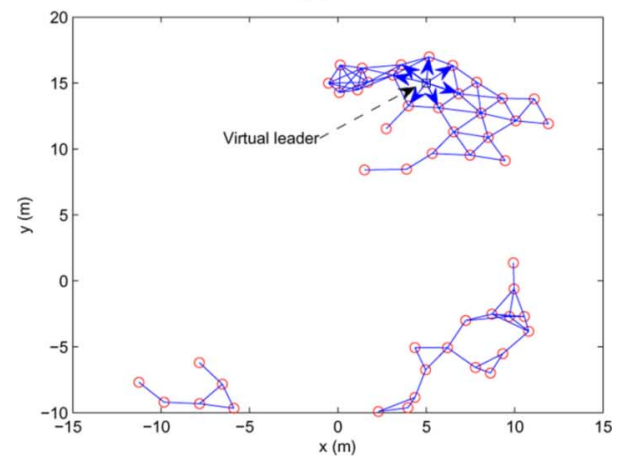

(b)

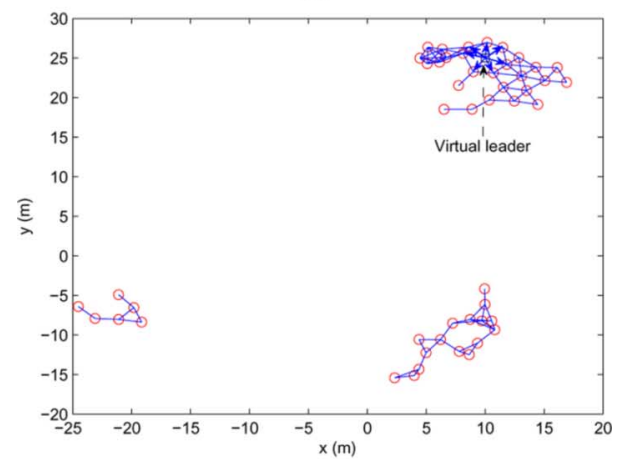

(c)

Fig. 8. Distributed swarm tracking for 49 followers with a virtual leader using (30) in 2-D. The circles denote the positions of the followers while the square denotes the position of the virtual leader. An undirected edge connecting two followers means that the two followers are neighbors of each other while a directed edge from the virtual leader to a follower means that the virtual leader is a neighbor of the follower. (a) $t=0 \mathrm{~s}$. (b) $t=5 \mathrm{~s}$. (c) $t=10 \mathrm{~s}$.

$r_{0}(t)=[t-5,-5+10 \sin (\pi t / 25)]^{T}, \alpha=1$, and $\beta=1.5$. The trajectories of the followers and the virtual leader are shown in Fig. 3. The tracking errors of the $x$ and $y$ positions are shown in, respectively, Fig. 4(a) and (b). It can be seen from Fig. 4 that the tracking errors converge to zero in finite time. That is, all followers track the virtual leader accurately after a finite period of time as also shown in Fig. 3.

For distributed swarm tracking in the case of first-order kinematics, we choose $R=2.5, d_{i j}=2, \alpha=1$, and $\beta=3$. The partial derivative of the potential function is chosen as in (36). Using (11) for (1) in 2-D, Fig. 5 shows the consecutive snapshots of distributed swarm tracking for 48 followers with a virtual leader. The initial states of the followers are randomly chosen from the square box $[-5,15]^{2}$ and $r_{0}(t)$ is chosen as $[t, 5+10 \sin (\pi t / 25)]^{T}$. It can be seen that the followers ultimately stay close to the leader and the interagent collision is avoided.

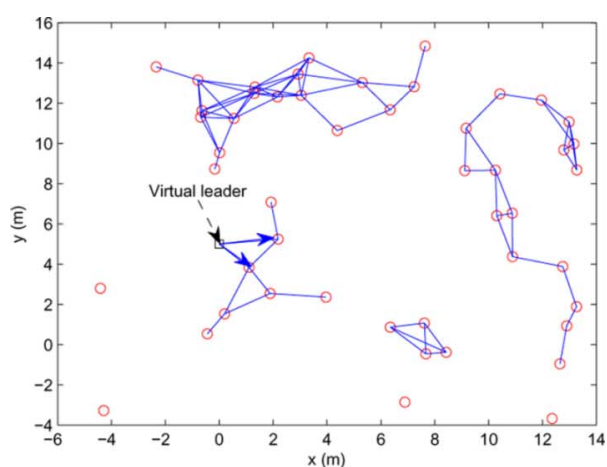

(a)

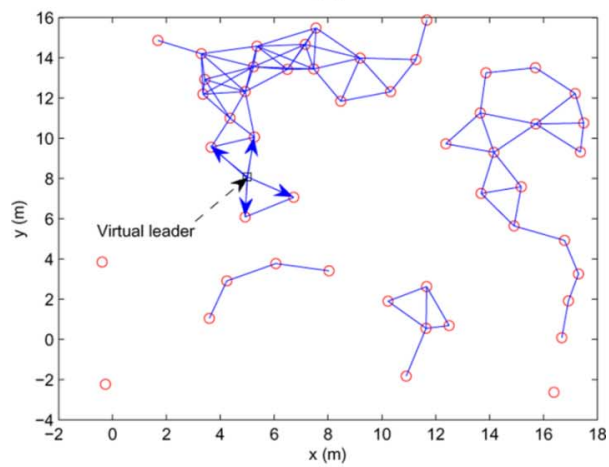

(b)

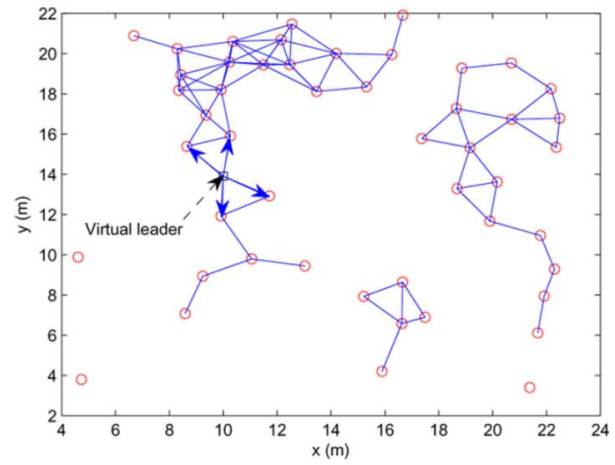

(c)

Fig. 9. Distributed swarm tracking for 50 followers with a virtual leader using (33) in 2-D. The circles denote the positions of the followers while the square denotes the position of the virtual leader. An undirected edge connecting two followers means that the two followers are neighbors of each other while a directed edge from the virtual leader to a follower means that the virtual leader is a neighbor of the follower. (a) $t=0 \mathrm{~s}$. (b) $t=5 \mathrm{~s}$. (c) $t=10 \mathrm{~s}$.

In the case of second-order dynamics, the network topology is chosen as in Fig. 2(b). It can be noted that the undirected graph $\mathcal{G}$ for all followers 1 to 6 is connected as well and the virtual leader is a neighbor of follower 1. Using (15) in 2-D, we choose $r_{0}(t)=[t, t+\sin (t)]^{T}, \alpha=1, \beta=5$, and $\gamma=0.1$. The trajectories of the followers and the virtual leader are shown in Fig. 6. The tracking errors of the $x$ and $y$ positions are shown in Fig. 7(a) and (b). The tracking errors of the $x$ and $y$ velocities are shown in Fig. 7(c) and (d). It can be seen from Fig. 7 that the tracking errors ultimately converge to zero. That is, all followers ultimately track the virtual leader as also shown in Fig. 6.

For distributed swarm tracking in the case of second-order dynamics, we choose $R=2.5, d_{i j}=2, \beta=5$, and $b_{i j}=1$ if vehicle $j$ is a neighbor of vehicle $i$ and $b_{i j}=0$ otherwise. The partial derivative of the potential function is chosen as in 


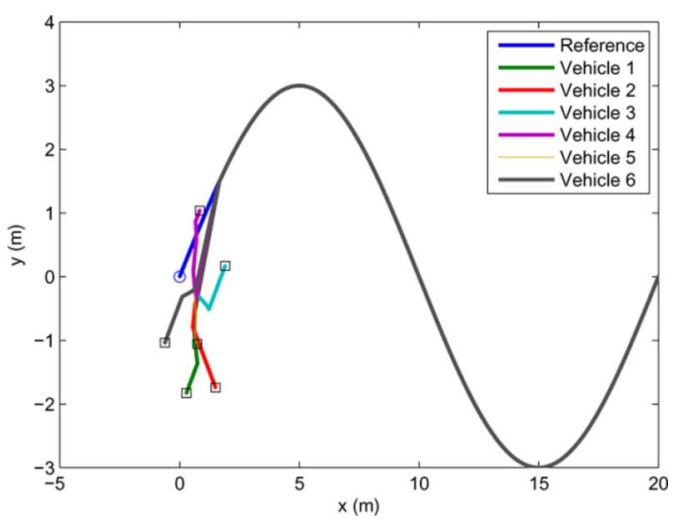

Fig. 10. Trajectories of the followers and the virtual leader using (26) in 2-D with connectivity maintenance mechanism. The circle denotes the starting position of the virtual leader while the squares denote the starting positions of the followers.

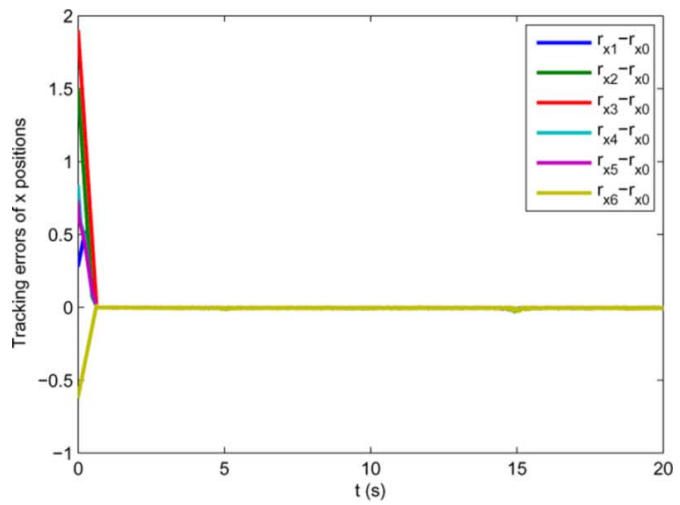

(a)

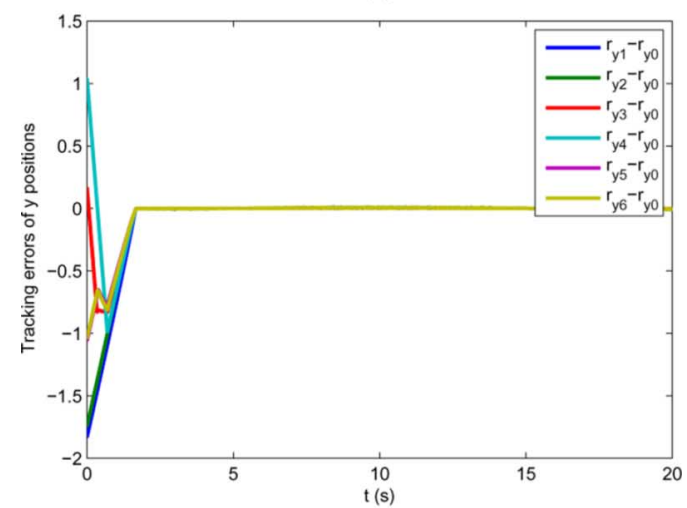

(b)

Fig. 11. Position tracking errors using (26) in 2-D with connectivity maintenance mechanism. (a) $x$ position. (b) $y$ position.

the case of first-order kinematics. In the case of a constant virtual leader's velocity, the initial states of the followers are randomly chosen from the square box $[-5,10]^{2}$ and $r_{0}(t)$ is chosen as $[t, 2 t+5]^{T}$. Using (30) for (14) in 2-D, Fig. 8 shows the consecutive snapshots of distributed swarm tracking for 49 followers with a virtual leader. In the case of a dynamic virtual leader's velocity, the initial states of the followers are randomly chosen from the square box $[-5,15]^{2}$ and $r_{0}(t)$ is chosen as $[t, 5+t+2 \sin (t)]^{T}$. We choose $\gamma=3$. Using (33) for (14) in 2-D, Fig. 9 shows the consecutive snapshots of distributed swarm tracking for 50 followers with a virtual leader. Due to the random choice of the initial states, the vehicles form separated subgroups initially. As a result, fragmentation appears in

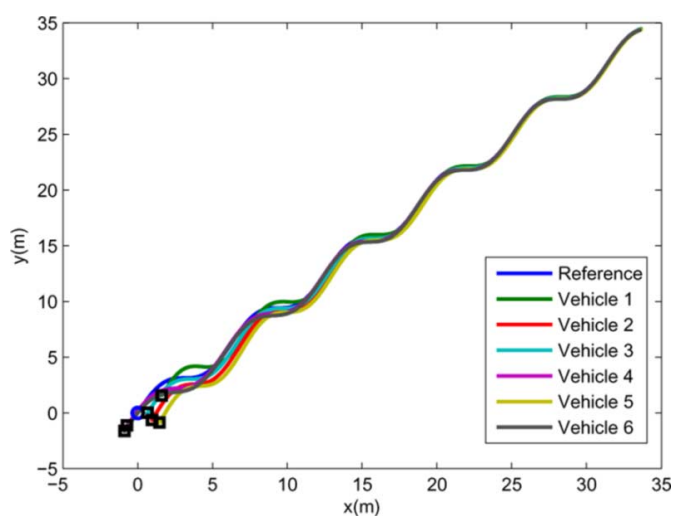

Fig. 12. Trajectories of the followers and the virtual leader using (23) in 2-D with connectivity maintenance mechanism. The circle denotes the starting position of the virtual leader while the squares denote the starting positions of the followers.

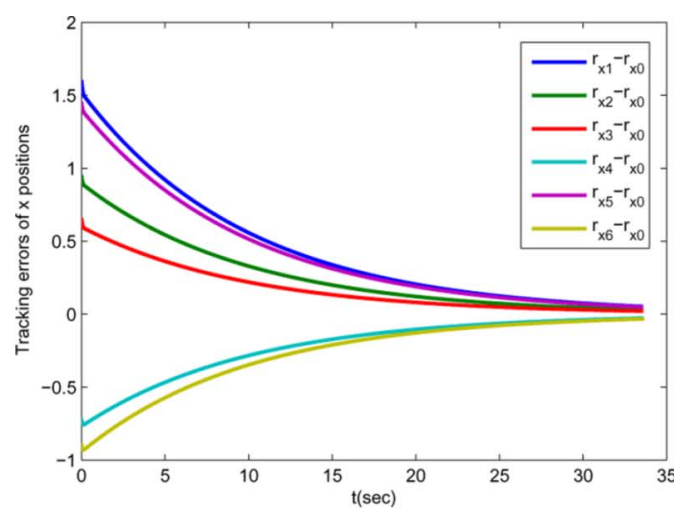

(a)

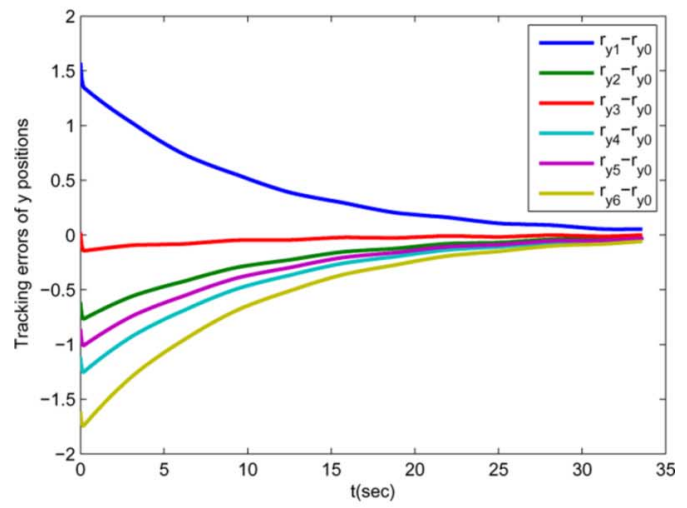

(b)

Fig. 13. Position tracking errors using (23) in 2-D with connectivity maintenance mechanism. (a) $x$ position. (b) $y$ position.

this case. However, for each subgroup, the relative distances of the followers and the virtual leader if the virtual leader is in the subgroup remain unchanged ultimately.

For distributed consensus tracking with the connectivity maintenance mechanism in Remark 4.7, we choose $R=3$ and $b_{i j}(t)$ according to Remark 4.7 with $(d / d t) b_{i j}(t)=$ $100\left\|r_{i}(t)-r_{j}(t)\right\| /\left(R^{2}-\left\|r_{i}(t)-r_{j}(t)\right\|^{2}\right)$ and $b_{i j}(0)=1$. Using (26) for (1) in 2-D with the connectivity maintenance mechanism in Remark 4.7, we choose $\alpha=1$ and $\beta=3$. Fig. 10 shows the trajectories of the followers and the virtual leader. The initial positions of the followers are randomly chosen from the square box $[-2,2]^{2}$ and $r_{0}(t)$ is chosen as $[t, 3 \sin (\pi t / 10)]^{T}$. The tracking errors of the $x$ and $y$ positions 


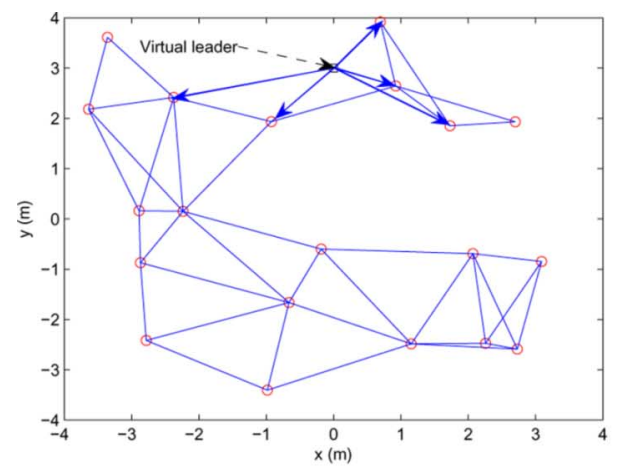

(a)

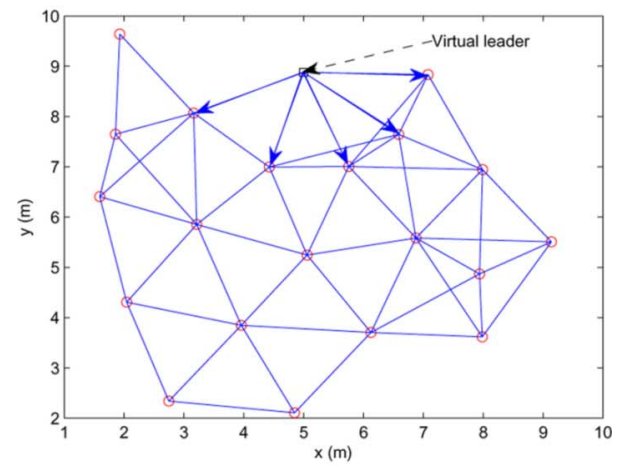

(b)

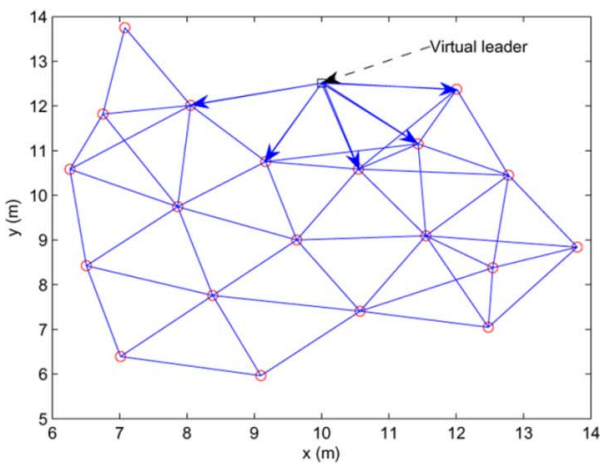

(c)

Fig. 14. Distributed swarm tracking for 20 followers with a virtual leader using (11) in 2-D in the presence of the connectivity maintenance mechanism in Remark 4.13. The circles denote the positions of the followers while the square denotes the position of the virtual leader. An undirected edge connecting two followers means that the two followers are neighbors of each other while a directed edge from the virtual leader to a follower means that the virtual leader is a neighbor of the follower. (a) $t=0 \mathrm{~s}$. (b) $t=5 \mathrm{~s}$. (c) $t=10 \mathrm{~s}$.

are shown in Fig. 11(a) and (b). It can be seen that the tracking errors ultimately converge to zero. That is, all followers ultimately track the virtual leader as also shown in Fig. 10. Using (23) for (14) in 2-D with the connectivity maintenance mechanism in Remark 4.7, we choose $\alpha=1, \beta=3$, and $\gamma=0.1$. Fig. 12 shows the trajectories of the followers and the virtual leader. The initial positions of the followers are randomly chosen from the square box $[-2,2]^{2}$ and $r_{0}(t)$ is chosen as $[t, t+\sin (t)]^{T}$. The tracking errors of the $x$ and $y$ positions are shown in Fig. 13(a) and (b). It can be seen from Fig. 13 that the tracking errors ultimately converge to zero. That is, all followers ultimately track the virtual leader as also shown in Fig. 12.

For distributed swarm tracking with the connectivity maintenance mechanism as in Remark 4.13, all parameters are

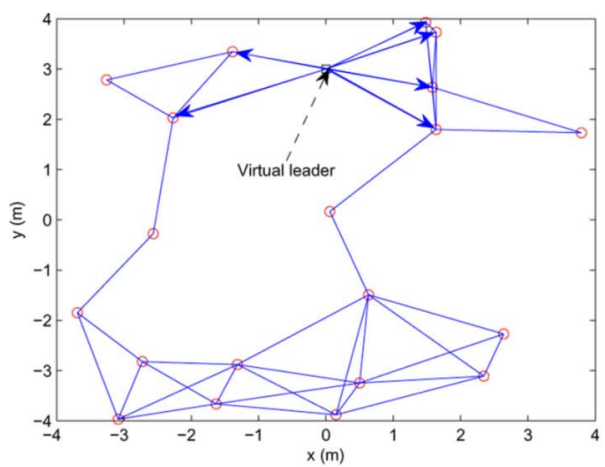

(a)

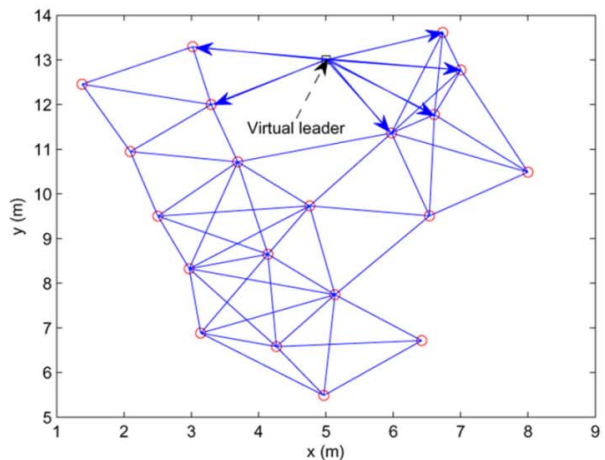

(b)

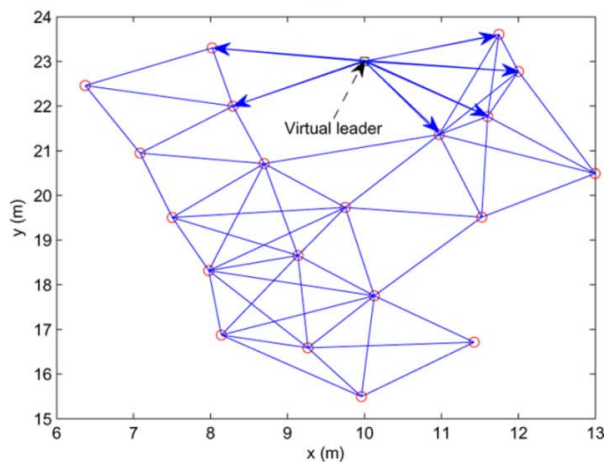

(c)

Fig. 15. Distributed swarm tracking for 20 followers with a virtual leader using (30) in 2-D in the presence of the connectivity maintenance mechanism in Remark 4.13. The circles denote the positions of the followers while the square denotes the position of the virtual leader. An undirected edge connecting two followers means that the two followers are neighbors of each other while a directed edge from the virtual leader to a follower means that the virtual leader is a neighbor of the follower. (a) $t=0 \mathrm{~s}$. (b) $t=5 \mathrm{~s}$. (c) $t=10 \mathrm{~s}$.

chosen the same as those for distributed swarm tracking without connectivity maintenance. When two followers are initially neighbors of each other or the virtual leader is initially a neighbor of some follower(s), the partial derivative of the corresponding potential function is chosen as (37). Otherwise, the partial derivative of the potential function is chosen as (36). In the case of first-order kinematics, the initial positions of the followers are randomly chosen from the square box $[-4,4]^{2}$ and $r_{0}(t)$ is chosen as $[t, 3+t+2 \sin (t)]^{T}$. Fig. 14 shows the consecutive snapshots of distributed swarm tracking for 20 followers with a virtual leader in 2-D with the connectivity maintenance mechanism in Remark 4.13. In the case of second-order dynamics with a constant virtual leader's velocity, the initial positions of the followers are randomly chosen from the square box $[-4,4]^{2}$ and $r_{0}(t)$ is chosen as $[t, 3+2 t]^{T}$. Fig. 15 shows the consecutive snapshots of distributed swarm 


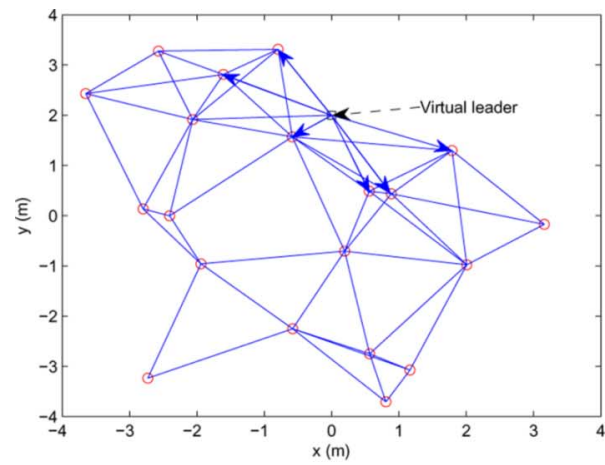

(a)

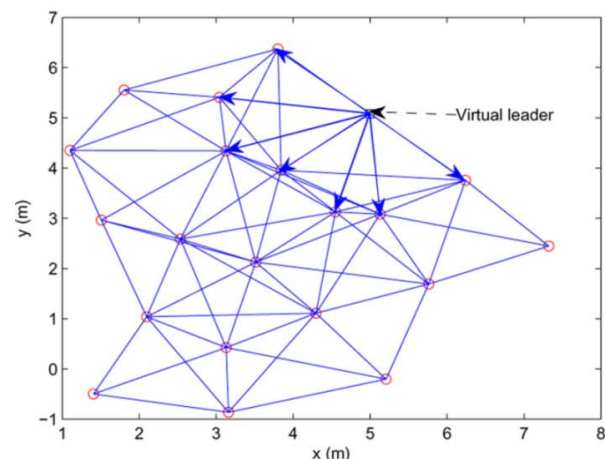

(b)

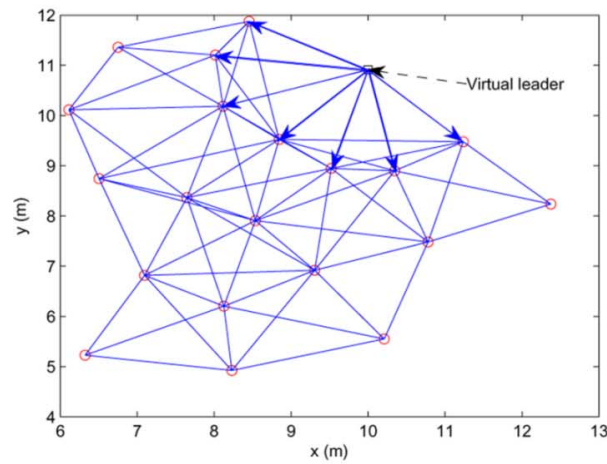

(c)

Fig. 16. Distributed swarm tracking for 20 followers with a virtual leader using (33) in 2-D in the presence of the connectivity maintenance mechanism in Remark 4.13. The circles denote the positions of the followers while the square denotes the position of the virtual leader. An undirected edge connecting two followers means that the two followers are neighbors of each other while a directed edge from the virtual leader to a follower means that the virtual leader is a neighbor of the follower. (a) $t=0 \mathrm{~s}$. (b) $t=5 \mathrm{~s}$. (c) $t=10 \mathrm{~s}$.

tracking for 20 followers with a virtual leader in 2-D with the connectivity maintenance mechanism in Remark 4.13. In the case of second-order dynamics with a varying virtual leader's velocity, the initial positions of the followers are randomly chosen from the square box $[-4,4]^{2}$ and $r_{0}(t)$ is chosen as $[t, 2+t+2 \sin (t)]^{T}$. Fig. 16 shows the consecutive snapshots of distributed swarm tracking for 20 followers with a virtual leader in 2-D with the connectivity maintenance mechanism in Remark 4.13. It can be seen that at each snapshot the network topology for the 20 followers is connected and the virtual leader is a neighbor of at least one follower because of the initial connectivity and the existence of the connectivity maintenance mechanism. Meanwhile, the relative distances of the followers and the virtual leader remain unchanged ultimately. In contrast to Fig. 5, Figs. 8 and 9 where the initially existing connectivity patterns might not always exist, the initially existing connectivity patterns in Figs. 14-16 always exist due to the existence of connectivity maintenance mechanism.

\section{CONCLUSION AND FUTURE WORKS}

In this paper, we studied a distributed coordinated tracking problem via a variable structure approach when there exists a dynamic virtual leader who is a neighbor of only a subset of a group of followers, all followers have only local interaction, and only partial measurements of the states of the virtual leader and the followers are available. For first-order kinematics, we proposed a distributed consensus tracking algorithm without velocity measurements and showed that distributed consensus tracking can be achieved in finite time. We then extended the result to derive a distributed swarm tracking algorithm in the absence of velocity measurements. For second-order dynamics, we proposed two distributed consensus tracking algorithms without acceleration measurements when the velocity of the virtual leader is varying and showed that the proposed algorithms guaranteed at least global exponential tracking. We then proposed a distributed consensus tracking algorithm and a distributed swarm tracking algorithm when the velocity of the virtual leader is constant. When the velocity of the virtual leader is varying, distributed swarm tracking was solved by employing a distributed estimator. For distributed consensus tracking (respectively, swarm tracking) problems, a mild connectivity requirement was proposed by adopting an connectivity maintenance mechanism in which the adjacency matrix (respectively, the potential function) is defined in a proper way. Several illustrative examples were presented to show the effectiveness of our algorithms. Future works include the study of distributed consensus tracking and swarm tracking algorithms in directed networks and finding the conditions of the control gains in a distributed manner.

\section{REFERENCES}

[1] Y. Cao and W. Ren, "Distributed coordinated tracking via a variable structure approach-Part I: Consensus tracking \& part II: Swarm tracking," in Proc. Amer. Control Conf., Baltimore, MD, Jun. 2010, pp. 4744-4755.

[2] N. E. Leonard, D. A. Paley, F. Lekien, R. Sepulchre, D. M. Fratantoni, and R. E. Davis, "Collective motion, sensor networks, and ocean sampling," Proc. IEEE, vol. 95, no. 1, pp. 48-74, Jan. 2007.

[3] R. M. Murray, "Recent research in cooperative control of multivehicle systems," J. Dynam. Syst., Meas., Control, vol. 129, no. 5, pp. 571-583, Sep. 2007.

[4] W. Ren and R. W. Beard, Distributed Consensus in Multi-vehicle Cooperative Control. London, U.K.: Springer-Verilag, 2008.

[5] Z. Qu, Cooperative Control of Dynamical Systems. London, U.K.: Springer-Verilag, 2009.

[6] F. Bullo, J. Cortes, and S. Martinez, Distributed Control of Robotic Networks. Princeton, NJ: Princeton Unive. Press, 2009.

[7] R. Olfati-Saber, J. A. Fax, and R. M. Murray, "Consensus and cooperation in networked multi-agent systems," Proc. IEEE, vol. 95, no. 1, pp. 215-233, Jan. 2007.

[8] W. Ren, R. W. Beard, and E. M. Atkins, "Information consensus in multivehicle cooperative control," IEEE Control Syst. Mag., vol. 27, no. 2, pp. 71-82, Apr. 2007

[9] Y. Hong, J. Hu, and L. Gao, "Tracking control for multi-agent consensus with an active leader and variable topology," Automatica, vol. 42, no. 7, pp. 1177-1182, Jul. 2006.

[10] Y. Hong, G. Chen, and L. Bushnell, "Distributed observers design for leader-following control of multi-agent networks," Automatica, vol. 44, no. 3 , pp. 846-850, Mar. 2008 
[11] Y. Cao, W. Ren, and Y. Li, "Distributed discrete-time coordinated tracking with a time-varying reference state and limited communication," Automatica, vol. 45, no. 5, pp. 1299-1305, May 2009.

[12] W. Ren, "Consensus tracking under directed interaction topologies: Algorithms and experiments,' IEEE Trans. Control Syst. Technol., vol. 18, no. 1, pp. 230-237, Jan. 2010.

[13] K. Peng and Y. Yang, "Leader-following consensus problem with a varying-velocity leader and time-varying delays," Physica A, vol. 388, no. 2-3, pp. 193-208, Jan. 2009

[14] R. Olfati-Saber, "Flocking for multi-agent dynamic systems: Algorithms and theory," IEEE Trans. Autom. Control, vol. 51, no. 3, pp. 401-420, Mar. 2006.

[15] H. Su, X. Wang, and Z. Lin, "Flocking of multi-agents with a virtual leader," IEEE Trans. Autom. Control, vol. 54, no. 2, pp. 293-307, Feb. 2009.

[16] H. Shi, L. Wang, and T. Chu, "Flocking of multi-agent systems with a dynamic virtual leader," Int. J. Control, vol. 82, no. 1, pp. 43-58, Jan. 2009.

[17] J. Yao, R. Ordonez, and V. Gazi, "Swarm tracking using artificial potentials and sliding mode control," J. Dynam. Syst., Meas., Control, vol. 129, no. 5, pp. 749-754, Sep. 2007.

[18] F. R. K. Chung, Spectral Graph Theory. Providence, RI: Amer. Math. Soc., 1997.

[19] D. Shevitz and B. Paden, "Lyapunov stability theory of nonsmooth systems," IEEE Trans. Autom. Control, vol. 39, no. 5, pp. 1910-1914, 1994.

[20] A. F. Filippov, Differential Equations with Discontinuous Righthand Sides. Amsterdam, The Netherlands: Kluwer Academic, 1988.

[21] B. Paden and S. Sastry, "A calculus for computing Filippov's differential inclusion with application to the variable structure control of robot manipulators," IEEE Trans. Circuits Syst., vol. CAS-34, no. 1, pp. 73-82, 1987.

[22] F. H. Clarke, Optimization and Nonsmooth Analysis. Philadelphia, PA: SIAM, 1990.

[23] H. G. Tanner, A. Jadbabaie, and G. J. Pappas, "Flocking in fixed and switching networks," IEEE Trans. Autom. Control, vol. 52, no. 5, pp. 863-868, May 2007.

[24] M. M. Zavlanos, A. Jadbabaie, and G. J. Pappas, "Flocking while preserving network connectivity," in Proc. IEEE Conf. Decision and Control, New Orleans, LA, Dec. 2007, pp. 2919-2924.

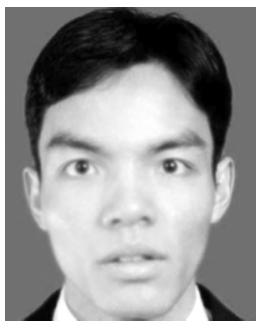

Yongcan Cao (S'07-M'11) received the B.S. degree from the Nanjing University of Aeronautics and Astronautics, Nanjing, China, in 2003, the M.S. degree from Shanghai Jiao Tong University, Shanghai, China, in 2006, and the Ph.D. degree from Utah State University, Logan, in 2010, all in electrical engineering.

His research interest focuses on cooperative control and information consensus of multiagent systems.

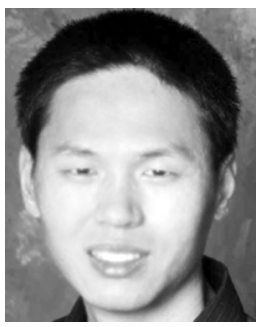

Wei Ren (S'01-M'04) received the B.S. degree in electrical engineering from Hohai University, Nanjing, China, in 1997, the M.S. degree in mechatronics from Tongji University, Shanghai, China, in 2000, and the Ph.D. degree in electrical engineering from Brigham Young University, Provo, UT, in 2004.

From October 2004 to July 2005, he was a Research Associate with the Department of Aerospace Engineering, University of Maryland, College Park. He was an Assistant Professor (August 2005 to June 2010) and an Associate Professor (July 2010 to June 2011) with the Department of Electrical and Computer Engineering, Utah State University, Logan. Since July 2011, he has been with the Department of Electrical Engineering, University of California, Riverside, where he is currently an Associate Professor. He is an author of the books Distributed Coordination of Multi-agent Networks (Springer-Verlag, 2011) and Distributed Consensus in Multi-Vehicle Cooperative Control (Springer-Verlag, 2008). His research focuses on distributed control of multiagent systems and autonomous control of unmanned vehicles.

Dr. Ren was the recipient of a National Science Foundation CAREER Award in 2008. He is currently an Associate Editor for the journals Automatica and Systems and Control Letters and an Associate Editor on the IEEE Control Systems Society Conference Editorial Board. 